\title{
Über logische und ethische Geltung.
}

Von Heinrich Rickert, Freiburg i. B.

I.

Das Problem.

Der Philosophie unserer Tage, besonders der an Kant orientierten, wird oft der Vorwurf gemacht, dass sie nur 'Theorie der Erkenntnis sei und sich damit auf ein zu enges Gebiet beschränke. Hierin liegt gewiss etwas Berechtigtes. Kant hat nie daran gedacht, die ganze Philosophie mit seiner neuen, transzendentalen Logik zu identifizieren, sondern seine Untersuchungen erstrecken sich auch auf das sittliche, das künstlerische und das religiöse Leben. Und, abgesehen hiervon, wird in der Tat nur die Geistesbetätigung den Namen der Philosophie verdienen, die den Menschen und seine Welt nach allen Seiten hin zum Gegenstande ihrer Erforschung macht. Trotzdem ist es nicht möglich, die Erkenntnislehre den iibrigen philosophischen Visziplinen so zu koordinieren, dass man Ethik oder Ästhetik obne Riicksicht auf logische Probleme treibt: Die wissenschaftliche Philosophie muss vielmehr mit allen ihren Teilen aus der Logik gewissermassen herauszuwachsen suchen, denn nur dann kaun sie sicher sein, auf wissenschaftlichem Boden zu stehen. Bei jedem Schritt in atheoretisches Gebiet hat sie erst ihr Verhältnis zum theoretischen festzustellen und dann ausdrücklich dafür zu sorgen, dass sie auch bei der Behandlung des Atheoretischen Theorie bleibt. Darin findet die Bevorzugung der Erkenntnislehre in der Philosophie unserer Zeit ihr woblbegründetes Recht.

Die Notwendigkeit einer erkenntnistheoretischen Besinnung tritt besonders dentlich zu Tage, wenn atheoretische Werte, wie z. B. die sittlichen, mit Rücksicht auf ihre Geltung zu philosophischen Problemen werden. Jede TVissensehaft, also auch die Ethik, nimmt die Form von Aussagen an, die gültigen Urteilsgehalt 
besitzen sollen. Handelt es sich um Seinsurteile oder Existenzialsätze in der weitesten Bedeutung des Wortes, so entsteht in Bezug auf das angegebene Problem keine Schwierigkeit. Ihr Geltungsgehalt ist, wenigstens der Absicht nach, unter allen Umständen rein theoretisch. Kommen dagegen sogenannte Werturteile in Betracht, dann bedarf es der Aufmerksamkeit darauf, ob und wie weit wir es noch mit theoretischen Geltungsbestandteilen zu tun haben.

Freilich wird man nicht überal! anerkennen, dass hier in Wahrheit ein Problem vorliegt. Manche glauben, die Frage nach der Wertgeltung leicht dadurch beseitigen zu können, dass sie Weiturteile aus der Wissenschaft überhaupt ausscheiden. Das wäre jedoch nur dann konsequent, wenn alle Werturteile atheoretische Bestandteile enthielten, und gerade das ist nicht zutreffend. Wird von einem Urteilsgehalt gesagt, dass er wahr sei, so ist danit gewiss ein Werturteil ausgesprochen, aber es geht nicht iiber die theoretische Sphäre hinaus. Doch vielleicht sieht man sich anch dadurch noch nicht veranlasst, Werturteile als wissenschaftliche Probleme anzuerkennen. Man kann meinen, sie liessen sich alle, soweit sie überhaupt Urteile sind, anf Seinsurteile zurïckführen. Dann würde z. B. in den Sätzen, dass ein Kunstwerk schön oder eine Willenshandlung gut ist, nichts über das Kunstwerk und die Villenshandlung ausgesagt, sondern lediglich behauptet, dass das urteilende Subjekt das Kunstwerk als schön und die Villenshandlung als gut werte, und auf die Konstatierung dieser Fakta, also auf Seinsurteile, käme es der Wissenschaft allein an. Dann müsste man aber, wenn man konsequent sein will, weiter gehen und behaupten, dass in den theoretischen Werturteilen ebenfalls nur die Tatsache konstatiert werden soll: das Subjekt hält ein logisches Gebilde für theoretisch wertvoll oder wahr. Diese Reduzierung auf einen reinen Seinsgehalt lässt sich jedoch nicht durchführen. Newne ich ein Urteil wahr, so will ich damit nicht nur sagen, dass ich es als wahr werte, sondern dass es unabhängig von meiner Wertung gilt, und ebenso ist es sicher wenigstens die Absicht des Urteilenden, wenn er ein Kunstwerk als schön oder eine Willenshandlung als gut bezeichnet, damit eine Geltung dieser atheoretischen Werte zu behaupten, die unabhängig von der Wertung des Subjekts besteht. Wie das theoretische Werturteil für einen Urteilsgehalt den Wert der Wahrheit, so nimmt das ästhetische oder das ethische Verturteil für ein 
Kunstwerk oder eine Willenshandlung die atheoretischen Werte der Schönheit und der Sittlichkeit als gültig in Anspruch, und damit ist ein Problem gegeben, das eine umfassende Philosophie nicht ignorieren darf.

Wie kommen wir dazu, in Form eines Urteils, also theoretisch, etwas über atheoretische Geltung auszusagen? Das ist nicht selbstverständlich. In der wissenschaf́tlichen Philosophie dürfen zunächst nur theoretische Werte als gültig vorausgesetzt werden, da sie von keinem theoretischen Standpunkt aus anzugreifen sind. Von den atheoretischen Werten, wie denen des sittlichen, des künstlerischen oder auch des religiösen Lebens, kann man das nicht sagen. Es muss daher gefragt werden, ob eine andere als theoretische Geltung von der Philosophie behauptet werden darf. Gibt es z. B. so etwas wie ethische Notwendigkeit und Allgemeingültigkeit auch für den wissenschaftlichen Menschen? Hierüber braucht jede Ethik Klarheit. Sie behandelt ein atheoretisches Wertgebiet in theoretischer Weise. Sie sucht nach Wahrbeit über die Sittlichkeit, nach dem theoretischen Wert über den ethischen Wert. So ist sie einerseits genötigt, die beiden Wertarten in engste Verbindung zu bringen, und darf sich doch andererseits nicht der Gefahr aussetzen, ihre Geltung zu vierwechseln. Dein aber entgeht sie nur, wenn sie die Begriffe des logischen und des ethischen Wertes auch mit Rücksicht anf das Verhältnis der ethischen zur logischen Geltung bestimnt. Deshalb versuchen wir im Folgenden zuerst, den ethischen Wert gegen den logischen soweit abzugrenzen, dass jede Vermengung ausgeschlossen ist, und sehen danu, welche Jerknüpfung zwischen logischer und ethischer Geltung trotz ihrer prinzipiellen Verschiedenartigkeit in einer wissenschaftlichen Ethik vorgenommen werden kann. Es soll damit ein Beitrag zur Logik der Ethik oder zur Bestimniung des wissenschaftlichen Charakters der Lehre vom Sittlichen gegeben werden. Wir werden sehen, dass aus den für die Trennung der Begriffe notwendigen Bestimmungen sich zugleich ihre unvermeidliche Verbindung ergibt. Doch beschränken wir uns dabei auf den denkbar umfassendsten oder rein formalen ethischen Wert und sein Verhältnis zur theoretischen Geltung und deuten den Inhalt des sittlichen Gutes nur an, wie das dem Charakter einer in der Hauptsache logischen Überlegung entspricht. 
II.

Theoretische Objektivität and praktische Subjektivität.

Bei dem Versuch der Abgrenzung und Trennung gehen wir selbstverständlich vom theoretischen Gebiet aus. Wie schon hervorgehoben, ist es das Urteil, das den logischen Wert der Wahrheit enthält. Aber dies Wort ist nicht eindeutig. Es kann darunter einmal der Satz verstanden werden, den wir als wahr meinen oder verstehen, und das andere Mal der Akt des Meinens oder Verstehens selbst. Ist das logische Urteil nun das grammatikalische oder das psychologische Gebilde? Wenn wir "Urteil" nur das nennen wollen, was im eigentlichen Sinn des Wortes ${ }_{n}$ wahr $^{\mu}$ seiu oder theoretisch „gelten " kann, so dürfen wir es weder mit dem Satz, noch mit dem Akt des Urteilens identifizieren, denn Wirklichkeiten sind als solche nicht gültig. Wahr ist vielmehr, streng genommen, nur der Urteilsgehalt, der von den Urteilsakten verstanden wird und an den Sätzen haftet, und der fällt mit keiner der beiden Wirklichkeiten zusammen. Das kann man schon daraus ersehen, dass er als gültige Wabrheit stets derselbe bleibt, wie verschieden auch die psychischen Vorgänge des Verstehens und die sprachlichen Ausdrücke der Sătze sein mögen. Der Urteilsgehalt also od̀er das guiltige Sinngebilde ist der eigentliche Gegenstand der theoretischen Philosophie. Der wirkliche Satz kommt für sie nur insofern in Betracht, als er einen unwirklichen Urteilsgehalt trägt, und die psychische Urteilsrealität ist nur insofern bedeutsam, als sie zu ihm Stellung nimmt, d. h., es handelt sich bei ihr nicht nm den psychischen Vorgang selbst, sondern um den ${ }_{n} \operatorname{Sinn}$, der ihr mit Rücksicht auf den logischen Gehalt innewohnt. Wir müssen daher von dem eigentlichen Urteilsgehalt, der unabhängig von allen Sätzen und psychischen Vorgängen gilt, und den wir deshalb auch den "transzendenten " logischen Sinn nennen können, einerseits das objektive Gut, an dem er haftet, und andererseits den subjektiven $\mathrm{Akt}$ der Stellungnabme mit dem ihm „immanenten “ Sinn sorgfältig scheiden. Der Urteilsgehalt ist von der Logik danu mit Rücksicht anf seine Form und seinen Inhalt zu untersuchen, wobei wir unter "Form" das theoretische Geltungsmoment in seiner begrifflichen Isolierung verstehen, durch welches der für sich logisch indifferente Inhalt in die logische Sphäre gehoben, also zum logisch gültigen Sinngebilde erst gemacht wird.

Wichtig für den folgenden Zusammenhang ist besonders dies. Das logische Wesen des subjektiven Urteilsaktes besteht für die 
Philosophie nicht in seiner psychologischen Struktur, sondern in der Bedeutung, die er für die Erfassung des transzendenten logischen Sinnes hat, und diese beruht darauf, dass er als Akt der theoretischen Entscheidung dem Inhalt die Form zuerkennt, oder Form und Inhalt als zusammengehörig bejaht. Ebenso ist das objektive theoretische Gut oder der logisch verständliche Satz nicht mit Rücksicht auf seine grammatikalische Struktur, sondern uur daraufhin zu untersuchen, wie er das transzendente Sinngebilde zum Ausdruck bringt. Wirkliche Sätze sind mit andern Worten dann allein logische Güter, wenn an innen unwirklicher Urteilsgehalt haftet, und der Akt des Snbjekts ist nur insofern wahr oder theoretisch wertvoll, als er ein objektives logisches Gut, also einen wahren Satz, meint oder versteht. Das logische Zentrum bleibt unter allen Umständen der Urteilsgehalt oder das transzendente Sinngebilde, das sich an den logisch wesentlichen Objekten, den Sätzen findet, und der subjektive Akt wird vollends' ausschliesslich durch seine Stellung zu ihm und dem theoretischen Gut logiscb bedeutsam. Vom objektiven Gut aus strahlt also der theoretische Wert in das subjektive Verhalten gewissermassen hinein.

Diese Gliederung des Gegenstandes der Logik in eine sub)jektive und eine objektive Sphäre kann hier näher nicht begründet werden. Sie soll auch nur ermöglichen, das theoretische Gebiet in seinem Verhältuis zum atheoretischen, besonders zum ethischen Gebiet zu bestimmen, und es entsteht nun die Frage, wie weit im Sittlichen oder im Gegenstand der Ethik dieselbe Struktur, insbeșondere die Anseinanderlegung in objektire und subjektive Faktoren und die Abhäugigheit des subjektiven Terhaltens vom objektiven Gut mit Rücksicht auf die IVertgeltung zu konstatieren ist. Ehe wir jedoch dazu übergehen, wollen wir zunächst einmal die Frage für den Gegenstand der Ästhetik stellen, un durch Vergleich die allgemeine Struktur der. IVertgebiete vielleicht noch deutlicher hervortreten za lassen. Wir finden hier nämlich eine weitgehende Übereinstimmung mit dem Theoretischen. Einmal haben wir. das ästhetische Gut, d. h. das wirkliche Kunstwerk mit dem daran haftenden gültigen, also unwirklichen ästhetischen Gebalt, das dem wahren Satz mit seinem transzendenten logischen Sinn entșprieht. Auf der andern Seite steht das Verhalten des Subjekts mit seinem immanenten ästhetischen Sinn, das dem logischen Urteilsakt parallel gesetzt werden kann, und das auch hier nur vom objektiven Gehalt und Gut her zu deuten ist. 
Ferner können wir in diesem ästhetischen Sinugehalt, ebenso wie im theoretischen, Form und Inhalt scheiden, wenn wir Form wieder das nennen, was als abstraktes. Wertmoment den ästhetisch indifferenten Inhalt in die ästhetische Sphäie hebt, und auch hier muss sich ein bestimmtes Verhältnis zwischen Form und Inhalt feststellen lassen, das den spezifisch ästhetischen Charakter des vom Subjekt unabhängigen, am Kunstwerk haftenden Sinngebildes bestimmt. So zeigt sich, dass auf theoretischem und ästhetischem Gebiet die Struktur des Gegenstandes der philosophischen Untersuchung im Wesentlichen dieselbe ist. Um beide Sphären von einander zu scheiden, braucht man nur das Wesen der theoretischen Form gegen das der ästhetischen abzugrenzen und sodann auf die Verhältnisse $\mathrm{zu}$ achten, in denen die verschiedenen Formen als begrifflich ablösbare Wertmomente zu den wertindifferenten Inhalten stehen. Die Hauptsache für uns ist jedoch das Gemeinsame: sowohl im theoretischen als im ästhetischen Gebiet liegt der Schwerpunkt auf dem Sinn oder Gehalt des objektiven Gutes, und das Yerhalten des Subjektes bekommt allein von hier aus seine ihm innewohnende Bedeutung für den Wert.

Gehen wir nun endlich zum ethischen Gebiet iiber, so scheint auch hier eine Gliederung in das Gut mit seinem Vertgehalt und das stellungnehmende Subjekt mit seinem immanenten Sinn vorgenommen werden zu müssen, wenn ron gültigen, objektiven Sinngebilden die Rede sein soll. Die Ethik ist, wie man sie auch sonst bestimmen mag, "praktische" Philosophie, d. h. sie untersucht den tätigen Menschen. Sittlich ist demnach immer ein Handeln, oder genauer: der ethische Sinn muss an einem Tun haften. Man könnte böchstens sagøn: nichts tun ist sittlich oder das ethische Ideal besteht in Passivität. Aber dies Nichtstun wäre dann auch eine Art des Tuns im weitesten Sinne des Worts. Demnach bezieht sich das Prädikat sittlich stets auf eine Willenshandlung, und gerade dieser Umstand scheint zunächst für die Gleichheit der Struktur des ethischen Wertgebietes mit dem theoretischen zu surechen. Das, was wir erreichen wollen, weun wir sittlich tätig sind, ist das ethische Gut, und es kann ethisch nur dann genannt werden, weun an ihm ein ethisches Sinngebilde haftet. Wir haben ferner ein ethisches Verbalten des Subjektes, das in der Terwirklichung des Gutes besteht, und es sieht nun so aus, als sei das Verhalten ethisch bedentsam wiederum nur wegen des Sinnes, den das erreichte objektive Gut trägt. Selbst- 
verstäudlich müssen wir dann in dem ethischen Sinngebilde wie im theoretischen Form und Inhalt von einander scheiden, denn es bedarf einer Form, die als abstraktes Wertmoment den ethisch indifferenten Inhalt in die ethischè Sphäre hebt, und es wird schliesslich das Verhalten des Subjektes nur dann ethisch sein, wenn es dem Inhalt die ethische Form beilegt, um so das ethische Gut hervorzubringen. Kurz, es scheint auch hier der ethische Charakter des subjektiven Verhaltens von dem ethischen Gut mit seinem objektiven Sinn abzuhängen.

Sehen wir jedoch genauer zu, so ergibt sich, dass im ethischen Reich die Gliederung, zunächst wenigstens, nicht in dieser Weise durchgeführt werden kann, und das weist darauf hin, dass noch tiefer liegende Unterschiede vorhanden sein müssen, als sie zwischen dem theoretischen und dem ästhetischen Gebiet bestehen. Ja, man kann geradezu sagen, es ist das Eigentümliche des Sittlichen, dass sich das Verhalten des. Subjektes von dem objektiven ethischen Gut nicht so ablösen lässt, wie das Verhalten des theoretischen Subjektes vom logischen Satz oder das Verhalten des ästhetischen Subjektes vom schönen Kunstwerk zu trennen war. Man braucht, um das einzuseben, nur zu fragen, was an der Willenshandlung den Namen des Ethischen verdient, oder woran der ethische Sinn haftet. Das ist nicht selbstverständlich. An jedem Tun sind zwei Seiten zu unterscheiden, der Ville im engereli Sinne als der „innerliche" Vorgang und die eigentliche Handlung mit ihren Wirkungen in der Aussenwelt. Sollte die Gliederung in eine objektive und eine subjektive Sphäre hier in derselben Weise vorgenomnen werden wie auf dem theoretischen Gebiet, so müsste der Wille nur das subjektive Verhalten darstellen und seine ethische Bedeutung erst von dem objektiven Erfolg her bekommen, an dem dann das unwirkliche ethische Sinngebilde mit seiner Geltung zu finden wäre. Gerade das aber scheint fraglich. Dürfen wir vom Willen sagen, er werde ethisch dadurch, dass er ethische Güter hervorbringt in der Weise, wie "ein Urteilsakt theoretisch sinnvoll dadurch wird, dass er einen wahren Satz mit seinem objektiven Sinn meint oder versteht?

Gewiss kann man von sittlichen Gütern reden, aber wenn nian das tut, so hat man dabei nicht den Erfolg im Auge, wie er für sich, d. h. ohne Beziehung auf den Willen des Subjekts sich darstellt. Das würde, konsequent zu Ende gedacht, zu Ergebnissen führen, die niemand meint, wenn er Handlungen sittlich nennt. 
Welche Erfolge wir in der Welt ausser uns haben, hängt nur zum Teil von uns ab, und daher ist es nicht möglich, uns für sie zur Verantwortung zu ziehen. Schon deshalb darf man von ihnen die sittliche Qualität der Handlung nicht herleiten, denn etwas, wofür wir nicht verantwortlich sind, ist auch nicht als Träger unserer Sittlichkeit anzusehen. Der Erfolg einer Willenshandlung ist also nie in dem Sinne ethisch, wie ein Satz wahr oder ein Kunstwerk schön ist. Nur der ge wollte Erfolg kommt in Betracht, ja der Wille oder die „Gesinnung", wie man sagt, ist allein das, was dem Handeln sittliche Bedeutung verleint, und zwar der von Absichten geleitete Wille, der weiss, was er will, und der auch kein blosser Wunsch ist, sondern ein Entschluss, der sich notwendig in ein Tun umsetzt. Das alles ist schon oft dargelegt, und das einfachste Beispiel muss von der Richtigkeit dieser Gedanken überzeugen. Sucht jemand mit Lebensgefahr einen Menschen zu retten, so wird die Handlung nicht sittlicher, wenn sein Bemühen gelingt, nicht weniger sittlich, wenn der Erfolg aushleibt. Auch an ein Wort von Mephistopheles können wir denken. Er nennt sich einen Teil von jener Kraft, die stets das Böse will und stets das Gute schafft. Er hat also immer gute Erfolge. Hinge seine sittliche Qualität davon ab, so müsste er die Verkörperung des sittlichen Prinzipes sein, und doch zweifelt niemand daran, dass er das böse Prinzip darstellt, weil eben sein Wille böse ist. Höchstens einige Ethiker werden das bestreiten, und auch sie nur dann, wenn sie Theorie treiben. Im Leben urteilen sie wie wir, und in diesem Falle ist die Meinung des "gesunden Menschenverstandes" nicht ganz so unwichtig wie sonst in der Philosophie.

Von sittlichen Erfolgen sollte man also eigentlich nicht reden. Wem es schwer wird, dies einzuseben, der macht sich nicht klar, dass es wertvolle Erfolge gibt, obne dass sie darum auch sittlich wertvoll sind. Wir können die Werke eines Menschen sehr hoch schätzen, und wir brauchen ihm trotzdem keine sittliche Grösse zuzusprechen, so lange es uns fraglich ist, ob das, was er getan hat, einem sittlichen Willen entsprang. Sollte man finden, dass dadurch das Wort "sittlich“ eine zu enge Bedeutung erhält, so ist dagegen selbstverständlich nichts zu sagen. Aber darauf kommt es bier nicht an. Zweifellos hat das Wort unter anderen auch die angegebene Bedeutung, und den von ihr gemeinten Wert allein wollen wir hier mit Rücksicht auf seine Geltung untersuchen. 
Mit diesen Überlegungen ist ein Unterschied des etbischen Gebietes vom theoretischen klar gestellt, der für unser Problem von Wichtigkeit sein muss. Es kann nicht von einem objektiven ethischen Gut aus das sittliche Verhalten des Subjekts den ethischen Charakter bekommen, wie von einem objektiven theoretischen Gut aus das urteilende Verhalten des Subjekts theoretisch zu deuten ist, sondern nur an. subjektiven Willen vermag das ethische Sinngebilde $\mathrm{zu}$ haften, und so scheinen wir von einem objektiven ethischen Gut in der Weise, in der wir vom wahren Satz als von einem theoretischen oder vom Kunstwerk als von einem ästhetischen Gut gesprochen haben, nicht reden zu dürfen. Der Platz dafür bleibt entweder leer, oder höchstens ist von der ethischen Gesinnung des Subjektes aus der Wert des Sittlichen auf den objektiven Erfolg zu übertragen, so dass hier, genau ungekehrt wie auf dem logischen und dem ästhetischen Gebiet, nicht der objektive Gehalt den Sinn des Subjektaktes bestimmt, sondern der subjektive Sinn des Verhaltens massgebend für den objektiven Wert des Gutes wird. Der theoretischen Objektivität scheint eine praktische Subjektivität gegenüber zu treten, welehe die Geltung des ethischen Wertes von vornherein auf ein anderes Niveau als das des theoretischen Wertes herabdrückt. So wird vollends klar, wie notwendig es ist, zu fragen: in welchem Sinne darf man in der Wissenschaft von objektiver ethischer Geltung sprechen?

III.

Der a u tonome Wille.

Die Antwort lässt sich mit den bisher vollzogenen Bestimmungen nicht geben. Um den ethischen Wert mit dem theoretischen in Hinsicht seiner Geltung vergleichen .zu können, gehen wir daher noch etwas auf seine Beschaffenheit ein und suchen dann auch den Begriff des ethischen Gutes zu gewinnen, das bisher ganz problematisch blieb. In erschöpfender Weise ist das hier natürlich nicht möglich. IVir werden einige unbewiesene Voraussetzungen machen und unser Ergebnis in dem Sinue einschränken mïssen, dass es nur bei Geltung dieser Voraussetzungen richtig ist. Doch dürfte in ihnen nicht allzuviel stecken, was heute nicht fast jede wissenschaftliche Ethik, implicite wenigstens, zugibt. Im Allgemeinen handelt es sich auch dabei wieder um sehr bekannte und fast selbstverständliche Gedanken, aus denen wir nur das für 
unsern Zusammenhang Unentbehrliche soweit hervorheben, dass später auf Grund dieser Bestimmungen das Verhältnis der theoretischen zur ethischen Geltung dem allgemeinsten Prinzip nach klar gelegt werden kann. Die etwas umständlichen Vorbereitungen dafür sind nicht zu vermeiden, wenn unsere Ansicht unzweidentig werden soll, denn gerade das Reich des Selbstrerständlichen ist Missverständnissen besonders stark ausgesetzt.

Wie also haben wir den ethischen Wert zu denken? Bisher wissen wir nur, dass sittlich der Wille ist, und zwar der bewusste, absichtlich verfahrende Wille, der sich notwendig in Handlungen umsetzt. Wessen muss nun der Wille sich bewusst sein, damit er sittlich wird? Stellen wir die Frage so, dann liegt die Antwort nahe. Wir werden nur den Menschen sittlich schätzen, der das will und tut, was er für das „Richtige" hält, d. h. wovon er glaubt, dass er es tun soll. Sittlich handeln wir mit auderen Worten dann, wenn die Pflicht unsern Willen bestimmt, unsittlich, wenn Rücksichten auf Vorteile oder Nachteile uns veranlassen, etwas zu tun, was gegen unser Pflichtbewusstsein verstösst. Wir dürfen danach den sittlichen Willen seinem allgemeinsten Sinn nach dem von der Pflicht geleiteten W'illen gleichsetz'n oder jedenfalls den Umstand, dass die Motive für den Willen im Bewusstsein des Sollens bestehen, für eine notwendige Bedingung alles sittlichen Wollens erklären. Im Grunde genommen ist auch camit nur etwas gesagt, was Niemand bestreiten kann. Wenn ich das will und tue, was ich meiner Überzeugung nach soll, so handle ich, wie ich soll, also sittlich in des Worts umfassendster Bedeutung. Trotzdem gibt es Richtungen in der Ethik, die gerade den Begriff des Sollens und der Pflicht nicht als entscheidend für die sittlichen Qualitäten einer Handlung anerkennen wollen. Der Grund dafür besteht jedoch nur darin, dass mit der angegebenen Begriffsbestimmung andere Gedanken verknüpft werden, die sorgfältig von ihr fernzuhalten sind. Deshalb bedarf es zur Verständigung noch einiger Worte.

Zunächst ist $\mathrm{zn}$ bemerken, dass hier nicht etwa gefragt wird, wie weit der Gesamtsinn unseres praktischen, persönlichen Leiens durch den Begriff des pflichtbewussten Willens sich erschöpfend deuten lässt, sondern dass es allein darauf ankommt, den spezifisch ethischen Charakter des Willens festzulegen, und dass es im Übrigen vollkommen unentschieden bleibt, welche Rolle das Sittliche in der Totalität unseres Daseins zu spielen hat. Wir sind weit davon entfernt, für irgend einen „Moralismus" einzutreteu. Ja, 
es sei ausdrücklich hervorgehoben, dass es für das persönliche Leben gewiss noch andere Werte als den des pflichtbewussten Willens gibt.

Doch, auch abgesehen hiervon; pflegt man gegen unsern $\mathrm{Be}-$ griff Einwände zu erheben. Sittlich ist nach ihm der Wille, der dem Pflichtbewusstsein gehorcht, und gerade den Gehorsam möchte man aus der Bestimmung der sittlichen Handlung entfernen. Die Unterordnung unter ein Gebot steht einem freien Wesen nicht an. Sie verlangen, heisst eine Sklavenmoral verkünden. Nicht: ich soll, sondern: ich will, hat die Parole des wahrhaft sittlichen Menschen zu lauten.

Das klingt Vielen wohl einleuchtend, und doch wird damit ein falscher Gegensatz eingeführt, der auf einem Missverständnis des Begriffs vom Pflichtbewusstsein beruht. In der Tat: wir haben der Pflicht zu "gehorchen“, aber es ist ein eigentümlicher Gehorsam, um den es sich dabei handelt. Wenn ein noch unerzogenes Kind seinem Vater gehorcht; so kommt es gewiss oft vor, dass es folgt, nur weil der Vater es will. Dann ist der befehlende Wille ein anderer als der gehorchende, und dieser Gehorsam kann den Willen allerdings nicht sittlich machen. Wer dagegen der Pflicht gemäss handelt, der gehorcht, wie man auch sagt, dem Gewissen und dann nicht einem Andern, sondern sich selbst. Der befehlende Wille fällt dann also mit dem gehorchenden zusammen. Danach ist der Gehorsam, den man des freien Menschen mit Recht für unwürdig hält, nur dort zu finden, wo das Pflichtbewusstsein noch nicht den Willen bestimmt. Unfrei ist gewiss, wer einem Andern gehorcht gegen den eigenen Willen. Aber unfrei ist auch, wer überhaupt von keinem Imperativ, sondern nur von Wünschen und Launen beherrscht wird. Wenn sittlicher Gehorsam Selbstgehorsam ist, dann bedeutet Sittlichkeit so viel wie Selbstgesetzlichkeit oder Autonomie. So muss man einsehen, wie falsch die Behauptung ist, das Pflichtgebnt erniedrige den Menschen, und es sei die Gleichsetzung von sittlichem und pflichtbewusstem Willen Sklavenmoral. Genau das Gegenteil ist richtig. Der pflichtbewusste Wille erhöht den Menschen zum Herrn, dẹnn er macht uns zum Herrn auch über uns selbst und befreit uns von jeder sittlichen Unfreiheit. Kurz, pflichtbewusst handeln, heisst nichts anderes als handeln, wie man es nach freier Überzeugung für gut hält, und keinen andern Richter anerkennen als die Stimme des. Gewissens in der eigenen Brust. 
Doch vielleicht wird diese Deutung Widerspruch von anderer Seite hervorrufen. So verstanden, kann man sagen, führt das Pflichtbewusstsein zur Aufhebung aller Sittlichkeit. Autonomie ist nichts als Zügellọsigkeit und Willkür. Gehorcht man nur sich selbst, so hört damit der Gehorsam, der diesen Namen verdient, ja die Anerkennung jedes Gebotes überhaupt auf. Das muss gerade durch die Trennung der zwei verschiedenen Arten des Gehorsams deutlich werden. Ist es denn unter allen Umständen unsittlich, einem Andern zu gehorchen? Kann nicht vielmehr gerade dies eine sittliche Notwendigkeit sein? Es gibt keine Sittlichkeit, ohne dass der Wille an einen Imperativ gebunden ist, dem er sich unterordnet.

Auch diese Einwände beruhen auf einem. Missverständnis. So gewiss der sittliche Mensch autonom ist, so gewiss bleibt er zugleich gebunden an sein Gewissen, und unter Umständen kann sehr wohl auch der Gehorsam gegen Andere inm Pflicht sein, sobald er nämlich einsieht, dass er notwendig ist mit Rücksicht auf ein von ihm anerkanntes Gut. Dann wird er sich freiwillig dem Andern unterordnen und gerade dadurch, dass er ihm gehorcht, sich selbst gehorchen. Der Willkür und Zügellosigkeit ist die richtig verstandene Autonomie ebenso entgegen gesetzt wie der Sklavenmoral. Sie ist zur Bestimmung des sittlichen Wertes deswegen geeignet, weil in ihr die beiden Bestandteile enthalten sind, auf die es beim Sittlichen ankommt, das freie Wollen und das Sollen, das individuelle Selbst und das darüber stehende Gebot. Darin steckt nur scheinbar ein Widerspruch. Wollen oder Sollen ist eine falsche Alternative. In Wahrheit kommt es auf die Vereinigung des Individuellen mit dem Überindividuellen, auf das freie Wollen des Sollens in der Anto-nomie an. Darum ist dies Wort die beste Bezeichnung für den ethischen Wert. Wir sind sittlich, wenn wir dem Gebot folgen, das wir selbst uns gegeben haben, ohne von aussen her irgendwie gezwungen zu sein. Wir sollen nur wollen, was uns als unser Sollen gegenübertritt, und was wir ganz in unsern Willen aufnehmen können, ohne ihm zn widerstreben.

Doch, es ist nicht notwendig, dies weiter auszuführen. Es galt wie gesagt nur, längst Bekanntes vor Missverständnissen zu schützen, und es genügt für unsern $Z$ weck, wenn wir dies festkalten. Ethisch ist der Mensch, der will, was er soll, indem er einen Wert durch freie Entscheidung als gültig anerkennt. Dem

Kantstudien $\mathbf{X I X}$. 
ethisch indifferenten Willen wird so durch die Selbstgesetzgebung der ethische Charakter aufgeprägt, und wir können daher die Autonomie auch als die Form bezeichnen, die der Inhalt haben muss, um als sittlich sinnvoll zü gelten. Diese Form als das abstrakte Geltungs- oder Wertmoment hebt den Willen in die ethische Sphäre oder verleiht ihm die ethische Bedeutung, wie die theoretische Form den Inhalt theoretisch sinnvoll macht, die ästhetische Form inn künstlerisch gestaltet. Damit tritt die Freiheit als die .formale Sittlichkeit der formalen Wahrheit und der formalen Schönheit als besonderer Wert gegenüber, und es müssen sich dementsprechend auch die inhaltlich erfüllten ethischen Sinngebilde von den inhaltlich erfüllten theoretischen und ästhetischen scheiden lassen. Sinngebilde, die in der Form der Autonomie stehen, nicht der psychische Vorgang des Wollens, der nur ihr Träger ist, sind der eigentliche Gegenstand der Ethik, und in ihrer Geltung steckt das ethische Problem.

\section{IV:}

Das ethische Gut.

Doch auch das genügt nicht, um diese Geltung in ihrem Verhältnis zur theoretischen zu begreifen, sondern es bleibt noch die Aufgabe, ausser dem Begriff des ethischen Verhaltens den Begriff des ethischen Gutes zu gewinnen und so neben das ethische Subjekt das ethische Objekt zu stellen. Oder sollte es überhaupt nicht möglich sein, von einem objektiven ethischen Gut zu reden? In einem gewissen Sinne enthält dieser Begriff in der Tat einen Widerspruch. Es gibt Güter, die der pflichtbewusste Mensch realisieren soll, sobald er sie als sinnvolle Güter erkannt hat, und er ist dann ethisch, wenn er das will, was er soll. Aber wir wiesen es ab, die Erfolge, auch wenn sie Güter sind, ethisch zu nennen, weil das Ethische eben nur am Willen hängt, und wir konnten daher bisher nur von logischen, äsihetischen oder irgend welchen andern Gütern reden. Wenn es demnach ethische Erfolge nicht gibt, so scheint es auch ethische Güter nicht geben zu können. Trotzdem hat dieser Begriff einen guten Sinn. Es kann der pflichtbewusste Wille oder der Träger der sittlichen Freiheit selbst zum Gut werden, das realisieren zu wollen, dann Inhalt der Pflicht sein muss. Oder mit andern Worten: es ist möglich, dass wir aus Pflicht einen autonomen Willen wollen, 
und dann handelt es sich um die Realisierung eines ethischen Gutes. Ja, es kann kein Zweifel sein, duss, wenn der freie Wille selbst als etwas zu Realisierendes auftritt, es notwendig Pflicht wird, ihn als wirklich zu wollen und dementsprechend $\mathrm{zu}$ handeln. Wir wollen daun subjektiv ethisch etwas objektiv Ethisches, oder ein Wille, der autonom die Autonomie will, ist zweifach sittlich, d. h. er ist einmal sittlich motiviert, er will aus Pflicht, und das, was er will, ist selbst das Sittliche, die Freiheit verwirklicht im pflichtbewussten Willen. Wenn dessen Realisierung gelingt, so können wir sogar von ethischen Erfolgen sprechen. Das hebt den Gegensatz von Erfolgs- und Gesinnungsethik nicht auf, denn der ethische Erfolg ist in diesem Falle ja die Realisierung der ethischen Gesinnung. IVir halten also, indem wir zum Begriff des ethischen Gutes fortschreiten und so den Durchbruch ins Objektive vollzieheu, an uuserm Begriff des Ethischen, der nur auf den Willen des Subjekts anwendbar ist, streng fest.

Dass die Scheidung in Gesinnung und Erfolg, in subjektives Verhalten und objektives Gut auch innerhalb des Ethischen notwendig ist, wird am dentlichsten, wenn ich z. B. bei der Erziehung es als Pflicht empfinde, den Willen eines andern Menschen pflichtberiusst zu machen. Falls dies gelingt, hat mein ethischer Wille einen ethischen Erfolg, der auch real von ihm verschieden ist. Freilich gibt diese Formulierung dem Prinzip einen einseitig altruistischen Charakter und nimmt auch sonst noch einiges vorweg, was wir von unsern Begriffen fern halten müssen. Trotzdem haben wir sie erwähnt, weil sie am geeignetsten ist, die Doppelheit in Ethischen klar zu stellen. Ihre Verallgemeinerung kanu keine Schwierigkeiten bereiteu. Wir brauchen bei dem Beispiel der Erziehung nur zugleich an Selbsterziehung zu denken. Danu fällt das altruistische Moment fort. Wir können also sagen: in doppelter Hinsicht ethisch ist jede IVillenshandlung, deren Erfolg ein ethischer Wille ist, und das ist auch insofern wichtig, als es nichts gibt, was so unbedingt Pflicht sein muss wie die Realisierung des ethischen Gutes. In allen andern Fällen kann man vielleicht $\mathrm{zw}$ eifeln, was gesollt ist. Tritt dagegen der sittliche IVille selbst als Ziel auf, dann ist jeder Zweifel an der ethischen Notwendigkeit seiner Verwirklichung ausgeschlossen. So gewinneu wir den Begriff des ethischen Gutes, ohne etwas Neues, erst besonders zu Begründendes $\mathbf{z a}$ den bisher entwickelten Begriffen hinzu zu nehmen, und wir konnten dies, weil mit der Beziehung 
des Wertes auf den Willen die Tendenz zur Realisierung notwendig verknüpft ist.

Was dieser letzte Schritt bedeutet, wird noch klarer werden, wenn wir daran denken, dass jeder ethische Wille, soweit wir ihn kennen, an einen Menschen gebunden ist, und dass wir diesen ethisch um so höher werten, je mehr sich sittliches Wollen und Handeln bei ihm nicht nur vereinzelt findet, sondern seinen ganzen Charakter beherrscht, so dass der pflichtbewusste Wille sich stets geltend macht, wenn es not tut. Wir können dann auch sagen: das höchste ethische Gut oder die denkbar vollkommenste Realisierung des Ethischen finden wir in einer vom Pflichtbewusstsein beherrschten, freien, autonomen Persönlichkeit. Dieser Begriff wird uns noch einmal am Schluss beschäftigen. Für unsere Zwecke reicht er zunächst in seiner einfachsten Gestalt aus als der eines Menschen, der in der Regel seine Pflicht tut und so in seiner Totalität autonom genannt werden kann. Die Persönlichkeit in diesem Sinne muss zum Zentralgut der Ethik als einer besonderen philosophischen Disziplin gemacht werden, wie der Satz mit seinem. wahren Sinn das Zentralgut der Logik, das Kunstwerk mit seiner Schönheit das Zentralgut der Ästhetik ist. Der Unterschied von theoretischer Objektivität und ethischer Subjektivität, von dem wir ausgingen, bleibt trotzdem streng gewahrt.

\section{V.}

Sachliche Kontemplation und persönliche Aktivität.

Wir müssen ihn sogar noch einmal betonen, denn er hängt mit Gegensätzen zusammen, die für die ganze Philosophie und ihre Einteilung massgebend sind, und es wird gut sein, dies ausdrücklich zu zeigen, damit die prinzipielle Seite unserer Bestimmungen noch mehr hervortritt. Alle objektiven Güter, an denen Werte haften, lassen sich in Sachen und Personen einteilen, und das subjektive Verhalten zu ihnen kann, wenn ihm. überhaupt ein Sinn mit Rücksicht auf Werte innewohnen soll, nur entweder Kontemplation oder Aktivität sein. Nun haben wir gesehen, dass der ethische Wert nicht an einer Sache, sondern. an einer. Person haftet, und ebenso ist klar, dass das ethische Verhalten nicht Kontemplation, sondern Aktivität bedeutet. Das wollen wir noch etwas weiter verfolgen, um die Unterschiede der beiden Wertgebiete in grössere Zusammenhänge zu bringen und. sie aus ihnen heraus zu verstehen. 
Achten wir zunächst auf den Gegensatz von Sache und Person, so begreifen wir, warum sachliche Güter, wie wahre Sätze und schöne Kunstwerke, stets real von dem Verhalten getrennt sind, das das theoretische und das ästhetische Subjekt zul ihnen hat. Nur dadurch wird das Gut zur "Sache“, dass es dem Subjekt als Objekt gegenübersteht, und damit ergibt sich das Auseinanderfallen in ein Objekt mit transzendentem und ein Subjekt mit immanentem Sinn als eine dem ganzen Gebiet des Sachlichen gemeinsame Notwendigkeit. Ebenso ist klar, warum das Subjekt hier als Person nicht in Betracht kommt, obwohl auch in der sachlichen Sphäre es faktisch immer eine Person ist. Es darf logisch und ästhetisch als Person nicht verselbständigt werden, sondern hat nur als blosses Subjekt zu gelten. Der immanente Sinn seines Verhaltens besteht ja auf diesem Gebiet allein darin, dass es sich der Sache oder dem transzendenten Sinn hingibt, also als Person verschwindet. Umgekehrt muss auf dem ethischen Wertgebiet, wenn wir dort überhaupt von einer Sache reden wollen, diese iluren Wert von der Person her empfangen. Das Gut, an dem der Sinn haftet, kaun daher real nicht als Objekt vom Subjekt getrennt werden, und die Person ist ferner hier niemals als blosses Subjekt zu betrachten. Da nur von ihr aus der Wert nach der Sache hinstrahlt, würde mit der Zerstörung des persönlichen Sinnes auf diesem Gebiet jeder Sinn überhaupt zerstört sein. So sehen wir vollends deutlich, in wie verschiedenen Sphären die theoretischen und die ethischen Werte liegen. Für die gesamte Wertphilosophie kann es einen prinzipielleren Gegensatz als den von persönlichen und sachlichen Gütern nicht geben.

Ein Beispiel wird seine Bedeutung für die Behandlung von einzelnen Problemen noch mehr hervortreten lassen. Nah verwandte Wertbegriffe, die leicht miteinander verwechselt werden, scheiden wir sicher, wenn wir sie ausdrücklich das eine Mal auf die Sache, das andere Mal auf die Person beziehen. Man sagt, dass jeder nur Wahres reden solle, und in dieser fiorm wird das vielleicht niemand bestreiten. Aber es macht doch einen wesentlichen Unterschied, ob man dabei den logischen, sachlichen oder einen ethischen, persönlichen Wert im Auge hat. Auf dem sachlichen Gebiete hat jeder gewiss nur wahre Urteile zu fällen, da an ihnen allein der logische Wert haftet. Die theoretische Persönlichizeit darf also nur die Pflicht kennen, sich als Subjekt mit ihren Urteilen in den Dienst der Wahrheitsverwirklichung zu stellen und Güter hervorzubringen, 
die nichts als wahr sind. Ihr Wert hängt duher auch ganz von den Erfolgen ab, die sie in dieser Hinsicht erzielt, d. h. ihre Bedeutung, die sie als theoretisches Subjekt hat, beruht ausschliesslich darauf, ob die von ihr getrennten sachlichen Güter, die sie hervorbringt, den Wahrheitswert tragen oder nicht. Sorgfältig hiervon zn scheiden ist jedoch die ethische Pflicht der Wahrhaftigkeit als persönlicher Wert. Zunächst kommt es hjer selbstverständlich nicht darauf an, ob der Mensch wirklich nur das sagt, was theoretisch wahr ist, sondern allein darauf, ob er das glaubt, was er spricht, oder es subjektiv für wahr hält, und seine IIandlungen, d. h. in diesen Falle seine Aussagen, werden in ihrem ethischen Wert nicht berührt, wenn sie die theoretische Wahrheit verfehlen. Aber las ist noch nicht alles. Haben wir uns einmal von dem theoretischen auf das ethische, also von dem sachlichen auf das persönliche Gebiet begeben, so steht es nicht mehr von vornherein fest, lass wir in jedem Fall die Pflicht haben, nur das zu sagen, was wir für wahr halten. Mit Hülfe des theoretischen Wahrheitswertes kann das jedenfalls nicht begründet werden, und es ist daher ganz ungerechtfertigt, den unter allen Umständen unsittlich zu nennen, der etwas sagt, wovon er weiss, dass es falsch ist. Es darf beim sittlichen Handeln niemals auf den sachlichen theoretischen IVert des Erfolges, sondern allein auf die persönliche Gesinnung und ihren Wert ankommen. Wo man jede bewusste Unwahrheit unsittlich nennt, hat man sachliche theoretische und persönliche ethische Werte in bedenklicher Weise miteinander verwechselt. Gewiss wird man vielleicht zeigen können, dass der. Mensch in Allgemeinen nicht ohne Not die Unwahrheit sprechen soll. Aber erstens lässt sich eine solche Begründung niemals mit Hülfe des sachlichen, theoretischen Wahrheitswertes geben, und ferner kann es unter Umständen sehr wohl vorkommen, dáss gerade ein gewissenhafter Mensch zur Unwahrheit sich ethisch genötigt sieht, nämlich weun er sich damit in den Dienst eines Gutes stellt, das zu realisieren, er für seine Pflicht hält, und dass er dann unsittlich handelt, wenn er trotzdem die Wahrheit spricht. Jedenfalls: etwas sagen, was theoretisch unwahr ist, und lügen in sittlich verwerflichem Sinne fällt in keiner Weise zusammen. Ein sachliches Gut, wie ein wahrer Satz, kann die sittliche Persönlichkeit nicht bessern, ein sachliches Übel, wie ein unwahrer Satz, sie nicht entwerten, während umgekehrt eine sittliche Persönlichkeit unter Umständen sehr wohl in der Lage ist, ein theoretisches Übel, wiẹ eine Un- 
wahrheit, zum Gut zu machen, ja eine unsittliche Persönlichkeit eventuell ein theoretisches Gut in ein Übel zu verwandeln vermag.

Doch kommt es hier nicht darauf an, das Verhältnis von sachlicher theoretischer Wabrheit und persönlicher sittlicher Wahrhaftigkeit in jeder Hinsicht zu klären. Wir haben dies viel umstrittene Beispiel nur herangezogen, weil sich an ihm besonders gut zeigen lässt, wie weit theoretische und ethische Werte auseinanderliegen, und wie dies leicht daraus zu verstehen ist, dass das eine Mal die Person, das andere Mal die Sache das Gut bildet, das als Träger des Sinngebildes in Betracht kommt.

Ebenso werden die Unterschiede zwischen den beiden Wertgebieten verständlich, wenn wir von dem Gegensatz der Aktivität zur Kontemplation ausgehen. Die Kontemplation ist nur dann sinnvoll, wenn das kontemplative Subjekt sich einem von ihm getrennten Objekt gegenüber befindet, auf welches es sich als seinen "Gegenstand" richtet, und von dem her sein Verhalten sachliche Bedeutung bekommt. So gehört es zum Wesen der Kontemplation, dass sie ein Objekt vom Subjekt ablöst. Der Abstand zwischen beiden kann auch aus diesem Grunde auf dem logischen und anf dem ästhetischen Gebiete niemals verschwinden, und das mystische oder "intuitive" Verhalten des Subjekts, das mit seinem Gegenstand zusammenfallen möchte, bleibt daher, wenn es Kontemplation sein soll, seiner sachlichen, unpersönlichen Bedeutung nach völlig problematisch. Es gibt keine sinnvolle Kontemplation ohne einen von ihr unabhängigen Gegenstand. Jedenfalls macht die theoretische oder wissenschaftliche Kontemplation alles, worauf sie sich richtet, zu einem rom Subjekt getrennten Objekt. Auch die Persönlichkeit nuss sie sich als Sache entgegenstellen, und nie kann das theoretische Subjekt mit dem Gut, an dem das transzendente Sinıgebilde haftet, sich in Eins setzen wollen. Ebenso haben wir zu einem Kunstwerk dann allein ein ästhetisches Verhältnis, wenn es etwas anderes ist als wir selbst. Sobald wir anfangen, uns mit dem künstlerisch dargestellten Gegenstund zu identifizieren, zerstören wir damit den ästhetischen Sinn, der an ihm haftet, so „reizvoll“ Vielen auch dies höchst unküustlerische Verhalten erscheinen mag.

Umgekehrt besteht das Wesen der Aktivität gerade darin, dass sie eingreift in das, was ihr gegenübersteht, oder dass sie das, was ihr Sinn geben soll, zu sich heran, ja in sich hinein zu ziehen sucht und nicht eher ruht, als bis jeder Abstand zwischen. 
Objekt und Subjekt vernichtet ist. Eine Handlung ist nur insofern „Handlung“, als sie jeden Gegen- und Widerstand überwindet und dann in einem Strom dahinfliesst, in dem es kein Aussen und kein Innen, kein Gespalten- und kein Gebrochensein zwischen dem Einen und dem Andern mehr gibt, wie es in jeder sinnvollen Kontemplation zu finden sein muss. Freilich, vor dem sittlichen Entschluss steht das Gebot dem Willen als etwas Fremdes gegenüber, aber so lange das währt, gibt es auch noch keine Autonomie. Der Wille duldet, wenn er ein sittlicher Wille ist, nicht, dass das Sollen von ihm getrennt bleibt, sondern indem er das Gebot will, das dem Handeln Sinn gibt, nimmt er es ganz in sich auf, um mit ihm zu verschmelzen. Erst mit der Lösung der Spannung setzt daher das Wollen ein, das in Wabrheit Aktivität ist, und es dauert dann so lange an, bis neue Gebote als Gegenstände auftauchen und von Neuem überwunden werden. So schäumt der Strom des Wollens am Widerstand des Sollens nur auf, um es in sich hinein- und mit sich fortzureissen. Freilich pflegt das Leben dafür zu sorgen; dass es an Widerständen dauernd nie fehlt, und dass insofern die Spannung niè endgültig gelöst wird. Ja, der aktive ethische Mensch wird es nicht anders haben wollen. Trotzdem besteht die einzelne ethische Handlung in der Überwindung des Sollens durch seine Aufnahme in den Willen, und so verstehen wir auch vom Begriff der Aktivität aus, dass der sittliche Wert nicht an einem rom sittlichen Verhalten getrennten Gut, sondern nur am sittlichen Verhalten selbst haftet. Subjekt und Ohjekt lassen sich hier uur dann real scheiden, wenn man das eine sittliche Subjekt dem andern gegenüberstellt. Einen festen, unüberwindlichen Gegenstand von der Art, wie die Kontemplation ihn braucht, um sinnvolle, „objektive“ Kontemplation zu sein, kann die ethisch sinnvolle Aktivität nie haben wollen, so sehr sie auch erst a n den Widerständen, nicht in einem glatten, in sich vollendeten Dahinfliessen ihr eigentliches Wesen zu entfalten vermag.

Kurz, es lässt sich sowohl vom Wesen der Sache aus verstehen, dass das sinnvolle Verhalten zu ihr kontemplativ sein muss, als auch aus dem Wesen der Kontemplation zu begreifen ist, dass sie sich auf eine Sache richtet, die dem subjektiven Verhalten entgegen steht. Und umgekehrt kann man sowohl vom Wesen der Persönlichkeit aus einsehen, dass ihr sinnvolles Verhalten Aktivität ist, als sich auch aus dem Wesen der Aktivität begreifen lässt, dass ihre Werte nur in Persönlichkeiten realisierbar sind. Damit 
haben wir die Besonderheiten der ethischen und der theoretischen Werte in einen grösseren Zusammenhang eingeordnet und mit dem Gegensatz der persönlichen Aktivität zur sachlichen Kontemplation in notwendige Verbindung gebracht.

\section{VI.}

\section{Logische und ethische Form.}

Schliesslich wird von hier aus noch ein eigentünlicher Unterschied deutlich, der das Form-Inhalt-Verhältnis auf dem persönlichen ethischen Gebiet von dem auf dem sachlichen logischen trennt. Wenn die Bedentung der logischen und auch der ästhetischen Form darin besteht, dass sie einen Inhalt objektiviert oder zur Sache macht und ihn so dem Subjekt gegenüberstellt, so muss der Inhalt zugleich in seiner Selbständigkeit erhalten bleiben, und die Form kann ihn nur umschliessen wie ein Gefäss. Es gibt kein Kunstwerk mit ästhetischem Sinn, in dem alles Form ist, sondern stets zeigt sich ein Inhalt so gestaltet, dass er auch als Inhalt ästhetisch wirkt, und ebenso ist in den abstraktesten theoretischen Begriffen ein inhaltlicher Faktor neben der Form aufzufinḋen, der als solcher alogischen Charakter trägt. Nicht nur ein "Inhalt überhaupt“, den man noch zum formalen Begriff des Gegenstandes überhaupt rechnen kann, sondern ein besonderer Inhalt des Inhalts ist erforderlich, wenn irgend "etwas" erkannt werden soll. Nennt man daher im Theoretischen die Form das Rationale, so gibt es keine theoretischen Gegenstände ohne ein irrationales, ron der logischen Form nur umgebenes und insofern getrenntes Element. Das Verhältnis von Form und Inhalt kommt hier, wie früher gesagt wurde, in dem Gedanken des Zusammengehörens zum Ausdruck, ohne den von theoretischer Geltung nicht gesprochen werden kann, und schon dies schliesst notwendig ein nie ganz zu überwindendes Auseinander ein. Beim ästhetischen Sinn fällt freilich das Zusammengehören fort. Der ästhetische Zustand ist gerade dadurch charakterisiert, dass Form und Inhalt als fragloses, atheoretisches Ineinander angeschaut werden können, und wenn wir trotzdem von der Form sagen, sie gehöre zum Inhalt, so sind wir damit aus dem ästhetischen Verhalten schon in das Urteilen über den ästhetischen Sinn gekommen und müssen daher den Gedanken des Zusammengehörens auf Rechnung dieses theoretischen Verhaltens setzen. Im künstlerischen bilden 
also Form und Inhalt im höheren Grade eine Einheit als im logischen Sinn. Dennoch ist auch hier ein Zusammen in der Weise zu konstatieren, dass der Inhalt der Form gegenüber noch seine Selbständigkeit bewahrt, und so muss es überall sein, wo sachliche Güter für die Kontemplation bestehen. Deshalb sagen wir, dass die Form hier den Inhalt nur umschliesst.

Im ethischen Sinngebilde dagegen, das am autonomen freien Willen haftet, ist von irgend einen Getrenntsein des Verbundenen oder von einer Fuge im Zusammengefügten keine Rede mehr. Wir wissen, der ethische Wille nimmt das Sollen restlos in sich auf, so dass beide zur Einheit verschmelzen, und das muss dann auch in der philosophischen Deutung des ethischen Sinngebildes, das der Wille trägt, seinen Ausdruck finden. Man könnte geradezu fragen, mit welchem Rechte man überhaupt noch von einer "Form“ im Unterschiede vom "Inhalt" spricht, wo es sich um das Verhältnis der Autonomie zum Willen handelt, und in ader Tat, wir benutzen diese Begriffe hier nur, um die verschiedenen philosophischen Wertprobleme sozusagen auf einen gemeinsamen Nenner zu bringen. Die Trennung in Form und Inhalt verdankt in Ethischen lediglich der Reflektion über den Sinn ihr Dasein, wie der Gedanke des Zusammengehörens im Ästhetischen ebenfalls nur durch das Urteil darüber entsteht, aber nicht zur Sache selbst gehört. Der frei handelnde Mensch wird in seiner Willenshandlung Willen und Freiheit nicht scheiden wollen. Der Wille selbst ist frei. Deswegen muss ausdrücklich hervorgehoben werden, dass auch das Form-Inhalt-Verhältnis auf dem ethischen Gebiet ein anderes Gepräge zeigt als auf dem logischen. Das lässt sich freilich nur mit Bildern ausdrücken, wie ja schon in den Begriffen der Form und des Inhaltes ein bildliches Moment steckt. Aber es wird wohl verständlich sein, wenn wir sagen, dass, während anf dem theoretischen, ebenso wie auf dem ästhetischen Gebiet die Form den Inhalt des Sinnes nur umschliesst und wie ein Gefäss ihm seine Selbständigkeit wahrt, im ethischen Sinngebilde die Form der Autonomie den Inhalt ganz durchdring $t$, so dass nichts an ihm ungefornit bleibt, und der Wille, seinem Sinne nach, in der Tat selbst frei genannt werden muss.

Dieser Unterschied wirft vielleicht das hellste Licht auf das Verhältuis von theoretischer und praktischer "Vernunft". Den theoretischen Rationalismus, der ein Erkennen durch die blosse Form voraussetzt, müssen wir ablehnen. Aus "reiner" theo- 
retischer Vernunft gibt es kein gegenständliches: Wissen. Auch die rationalsten Disziplinen kommen ohne ein irrationales, wenn man will, empirisches Moment im Gegenstand nicht aus. Dagegen könnten wir wohl von einem ethischen "Rationalismus" insofern sprechen, als die praktische Vernunft den Willen nur dann in Wahrheit frei macht, wenn sie ihn ganz zu ergreifen und zu durchtränken vermag, und von hier aus liesse sich dann vielleicht auch verstehen, warum die theoretische Philosophie sich dort so leicht rationalistisch gestaltet, wo sie, statt vom objektiven, sachlichen, transzendenten Urteilsgehalt, vom theoretischen Urteilsakt des Subjektes ausgeht. Sie fasst dann das lo gische Denken als ein "Erzeugen" des Gegenstandes oder als ein freies Tun auf, d. h. sie macht aus der "dualistischen", umscbliessenden, kontemplativen, theoretischen eine "monistische", durchdringende, aktive, praktische Vernunft. In einem gewissen Sinne hat das auch seine Berechtigung, und wir werden davon noch zu sprechen haben, wenn wir zu den Verbindungen gekommen sind, die zwischen dem theoretischen und dem ethischen Gebiet bestehen. Zunächst aber galt es, zu scheiden, und da war es wichtig, zu sehen, wie auch der Unterschied des Irrationalismus und des Rationalismus oder des Umschlossen- und des Durchdrungenseins des Inhalts von der Form sich sowohl mit Rücksicht auf den Gegensatz von Sache und Person als auch mit Rücksicht auf den von Aktivität und Kontemplation verstehen lässt. Die Sache wird nur dadurch zur Sache oder zum theoretischen Gegenstand, dass eine Form den Inhalt, der erkannt werden soll, zwar prägt, ihn in seiner Selbständigkeit aber zugleich bewahrt. Die Kontemplation bleibt nur dadurch Kontemplation, dass sie mit der Form einen Inha't zwar umschliesst, ihn aber zugleich als besonderen Inhalt vor sich hinstellt. So ist alles kontemplative Verhalten seinem Sinn nach ein „Vorstellen“ im Gegensatz zum aktiven Wollen. Und umgekehrt: die Persönlichkeit wird nur dadurch als Persönlichkeit ethisch sinnvoll, dass die Form der Freiheit sie nicht bloss umgibt, sondern ihren Willen in seinem innersten Wesen frei macht, und die sittliche Handlung ist nur dann wahrhaft Handlung, wenn nichts mehr sich ihr gegenüber befindet, was bloss "vor-gestellt" und nicht auch frei gewollt wird. 


\section{VII.}

\section{Der Wille zum Wissen.}

Jetzt ist das persönliche, aktive ethische Gut so weit von dem sachlichen, kontemplativen theoretischen getrennt, dass wir, ohne eine Vermengung befürchten zu müssen, auch nach den Beziehungen fragen können, die zwischen beiden bestehen. Damit kommen wir endlich zu dem eigentlichen Zweck unserer Überlegung. Wir wollten feststellen, in welchem Sinn die wissenschaftliche Ethik von einer Geltung sittlicher Werte sprechen darf. Die Verbindung des Ethischen mit dem Theoretischen kann nun aber von zwei Seiten vorgenommen werden, einmal vom Ethischen aus nach dem Theoretischen hin, und das andere $\mathrm{Mal}$ umgekehrt vom Theoretischen aus nach dem Ethischen hin, und beide Möglichkeiten müssen wir beachten. Die zweite Art ist für uns selbstverständlich die wichtigere, denn wir wollen ja wissen, was den theoretischen Menschen die ethischen Werte angehen, aber wir dürfen deshalb die erste Art nicht ganz ignorieren, und wir stellen daher zunächst die Frage: was hat der ethische Mensch mit den theoretischen Werten zu tun? Besteht zwischen dem Willen und dem Intellekt eine notwendige Verbindung, wenu wir uns anf den Standpunkt der sittlichen Persönlichkeit stellen?

Die Antwort ist auf Grund der vorher entwickelten Begriffe leicht zu geben. Nicht ohne Absicht hoben wir hervor, dass der sittliche Wille ein bewusster Wille sein muss, und im Bewusstsein der Pflicht sahen wir das ethische Kriterium. Schon hieraus kann man verstehen, dass auch die ethische Persönlichkeit ein Vissen, also ein theoretisches Gut braucht. Freilich ist das blosse „im Bewusstsein haben" des Sollens als solches noch kein theoretisches Verhalten, und vollends wird die sittliche Beurteilung niemals davon abhängig, ob der pflichtbewusste Mensch das, was ein Gut ist, und was er daher wollen soll, auch richtig erkannt hat. Der Irrende ist ebenso sittlich wie der Wissende. Aber es gehört trotzdem zum Begriff des ethischen Willens, dass der Wollende, wenn er seiner Sache nicht ganz sicher ist, wenigstens versucht, soweit er es vermag, nicht bei dem rein atheoretischen „Pflicht-

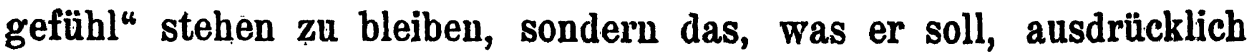
auch ins theoretische Wissen zu heben. Der Versuch dazu ist ebenfalls Sache des.Willens, nicht des Intellekts. So bleiben die Wertbegriffe nach wie vor streng geschieden. Den Willen zum Wissen können wir jedoch in der ethischen Persönlichkeit nicht 
entbehren, und damit ist trotz aller Scheidung , ein notwendiger Zusammenhang des Theoretischen mit dem Ethischen gegeben. Die Sprache hat ein charakteristisches Wort, um ihn zu bezeichnen, und sie dürfte dabei nicht nur unter dem Einfluss eines unberechtigten "Intellektualismus" stehen. Wir sagten schon, dass jemand dann allein einen sittlichen Willen hat, wenn er seinem "Gewissen" gehorcht. Das Gewissen ist das Bewusstsein von der Pflicht, und wir haben es als wollende Menschen zu einem Wissen von dem, was sein soll, auszugestalten. Insofern können wir Wissen und Wollen, theoretisches und ethisches Verhalten nicht trennen.

Selbstverständlich wird damit nicht jedem handelnden Menschen zugemutet, dass er sich mit Ethik bescbäftige. Die meisten würden mit Recht sagen, dass, auch wenn sie im Ungewissen über ihre Pflicht sind, sie wissenschaftlicher Überlegungen nicht bedürfen, um zu erkennen, was sie woilen sollen, und dass es unter Umständen sogar Pflicht sein kann, zu handeln, ohne dass man gecau das Richtige kennt, ja dass dies Handeln zu den schwersten Pflichten gehört. Wenn man sich aber einmal auf den wissenschaftlichen Standpunkt gegenüber dem sittlichen Leben gestellt hat, dann wird man es nicht vermeiden können, auch theoretisch darüber nachzudenken, was für den Menschen in Wahrheit Pflicht ist, und besonders wer sich über den Wert des pflichtbewussten Willens und das Gut der freien, autonomen Persönlichkeit klar geworden ist, muss fragen, was der Wollende zu tun hat, um ethische Werte in Gütern zu realisieren. Hierin steckt das relative Recht dessen, was man ethischen Intellektualismus neunt, und was man heute vielfach gänzlich abweist, um es durch einen ethischen Voluntarismus zu ersetzen. Indem wir hervorhoben, dass nur am Willen der sittliche Wert haftet, haben auch wir den Voluntarismus vertreten, und dabei muss es selbstverständlich bleiben. Aber das Wissen vom Richtigen, das Produkt des Intellekts, kann jederzeit und muss unter Omständen auf den Willen bezogen werden, und dann bekommt es auch ethische Bedeutung. Das ist hier von Wichtigkeit, denn schon das stellt das Verhältnis von sittlichem Wollen und theoretischem Wissen in einer Hinsicht klar. In dem angegebenen Sinne bleibt es richtig, dass die "Tugend" lehrbar ist. Der ethische Intellektualismus irrt jedenfalls nicht, wenn er rom sittlichen Menschen auch theoretisches Wissen verlangt. Unrecht hat er nur, wenn er den Schwerpunkt auf das 
Erkennen legt und alles atheoretische Verhalten gering schätzt oder gar meint, mit dem Wissen des Richtigen allein sei es getan, denn daraus werde das Wollen und Handeln sozusagen von selbst erfolgen. Er übersieht, dass ein Wissen ethisch noch uichts bedeutet, falls es nicht Motiv für einen pflichtbewussten Willen und eine ihm entspringende Handlung wird. Aber ebenso ist mit Nachdruck hervorzuheben, dass mit dem blossen "gedankenlosen" Wollen es auch nicht immer getan ist, sondern dass der ethische Mensch zugleich die Pflicht hat, zu wissen, was er soll, um so das Theoretische in den Dienst des Praktischen zu stellen.

Dementsprechend wird die Ethik als Wissenschaft weder für den reinen Intellektualismus noch für den reinen Voluntarismus eintreten können, sondern den Voluntarismus so gestalten, dass das relative Recht des Intellektualismus darin aufgehoben ist. Das gelingt ihr vom Begriff des theoretisch ausgebildeten Gewissens als des Wissens von dem, was sein soll. In ihm sind bereits ethische und logische Werte miteinander verknüpft, und zwar so, dass die theoretischen auf die praktischen gestützt erscheinen, insofern die ethischen hier die logischen fordern. Darin liegt nicht die geringste Wertvermengung, kein Übergreifen in ein fremdes Gebiet, sondern auch das ergibt sich notwendig aus dem Begriff des Ethischen als des pflichtbewussten Willens, und damit öffnet sich der Blick auf einen Weg, der vom ethischen Handeln aus in die Theorie des Praktischen oder in die Ethik als Wissenschaft. führt.

\section{VIII.}

Die Freibeit des Urteilens.

Aber das ist, wie gesagt, nur die eine Seite der Sache. Wichtiger ist für uns die Verbindung des Ethischen mit dem Theoretischen vom Theoretischen her. Ja; hier liegt die Entscheidung über unsere Hauptfrage: lassen die sittlichen Werte sich auf die logischen in der Weise stützen, dass in der wissenschaftlichen Ethik von ihrer Geltung gesprochen werden darf? Diesem Problem wenden wir uns jetzt zu. Der entscheidende Gedanke ist auch hier leicht aus den bereits entwickelten Begriffen abzuleiten. Wir brauchen nur das, was wir bisher getrennt haben, wieder aufeinander zu beziehen. Dann tritt der Zusammenhang zu Tage.

Bevor wir jedoch zum Positiven übergehen, wollen wir ausdrücklich sagen, in welchem Sinne man das Ethische mit dem Theoretischen nicht verknüpfen darf. Das ist schon deswegen 
notwendig, weil gerade bei Kant, dessen Gedankengänge zum Teil auch für uns massgebend waren, sich unzulässige Verbindungen finden. Er hat versucht, ethische Werte in ihrer Geltung dadurch zu stützen, duss er sie auf theoretische Werte zurückführte. So sollen gewisse Verbote deswegen ethisch gelten, weil jeder, der sie nicht anerkenut, sich damit in einen logischen Widerspruch verwickelt. Hierdurch kann für die wissenschaftliche Begründung ethischer Werte nichts geleistet sein, denn so gewiss ein ethischer kein theoretischer Wert ist, so gewiss bleibt es ein hoffnungsloses Unternehmen, aus dem logischen Satz des zu vermeidenden Widerspruches irgend ein ethisches Verbot herauszuklauben, das nicht schon vorher als gültig anerkannt war. Habe ich einmal ein Gut als ethisch wertvoll gesetzt, dann kann ich freilich zeigen, dass ich gewisse Handlungen nicht wollen darf, weil ich die von mir selbst gewerteten Güter dadurch zerstören, also inkonsequent sein würde, aber ohne diese Voraussetzung ist mit dem Satz vom Widerspruch in der Ethik nichts zu machen. Das muss um so schärfer hervorgehoben werden, als Kant durch Ausführungen wie die angedeuteten die Grösse seiner eigener Leistung, welche in der strengen Scheidung der verschiedenen Wertgebiete besteht, beeinträchtigt und Angriffe geradezu herausgefordert hat. Es darf nicht der Schein entstehen, als träfen Einwände gegen Einzelheiten, in denen Kant seinen eigenen Prinzipien untreu geworden ist, die Grundgedanken seiner Wertphilosophie.

Doch, auch abgesehen hiervon, sollte die Ethik, wenn sie von ethischer Geltung spricht, niemals darüber Unklarheit bestehen lassen, dass diese Geltung für alle Zeiten atheoretisch bleiben muss. Sie würde, wenn es ihr gelänge, die sittlichen Werte in theoretische zu verwandeln, nicht mehr Ethik sein, sondern dadurch zur Logik werden. Ethik aber ist nie Logik, auch nicht Logik des Wollens. MIan darf überhaupt nicht meinen, es liesse sich die Geltung ethischer Werte irgendwie "beweisen." Ein Wert, der durch Berveise Geltung bekommen hätte, wäre kein ethischer Wert mehr, sondern ein logischer. Nie Ethik wird gerade dadurch Wissenschaft, dass sie den ethischen Wert in seiner atheoretischen Eigenart charakterisiert und vor allen Verwechslungen mit theoretischen Werten schützt. Falls daher die Ansicht, dass ethische Werturteile nicht in die Wissenschaft gehören, nur besagen soll, dass ethische Werte nicht theoretisch gelten können, so ist dagegen nicht das Geringste einzurvenden. Zugleich aber wird man doch 
bezweifeln dürfen, ob durch solche Selbstverständlichkeiten sich das Problem einer Ethik als Wertwissenschaft abweisen lässt.

Ganz unabhängig von diesen Erwägungen besteht nämlich die Frage, ob es nicht möglich ist, die Autonomie als einen Wert zu verstehen, dessen Gültigkeit auch der theoretische Mensch anerkennen muss, obwobl sie stets ein atheoretischer Wert bleibt. Sollte das gelingen, so wäre damit alles geleistet, was von einer wissenschaftlichen Ethik mit Rücksicht auf die Begründung ihres Grundwertes verlangt werden darf. Dieser besässe dann zwar gewiss keine logische Geltung, die er niemals haben kann, so lange er ein ethischer Wert ist, aber er wäre trotzdem gültig auch für den theoretischen Menschen, und daher brauchte man nicht mehr zu fürchten, dass die Ethik mit unbegründeten Werturteilen uñter das Niveau der Theorie herabsteigt, wenn sie ausdrücklich die Autonomie als Wert bejaht. Wir versuchen also, eine Verbindung zwischen theoretischer und ethischer Geltung aufzuzeigen, die von der Art ist, dass sie dem ethischen Wert eine auch vom theoretischen Standpunkt nicht anzutastende Notwendigkeit verleiht und ihn damit über alle unwissenschaftliche Willkür hinaushebt.

Wie aber soll es zu einer solchen Verbindung kommen? Solange wir nur daran denken, dass der transzendente theoretische Sinn sich an einer Sache findet oder einen Gegenstand der Kontemplation bildet, in dem die Form als Wert den Inhalt umschliesst, und dass im Gegensatz dazu der ethische Sinn allein dem subjektiven Verhalten der aktiven Persönlichkeit zukommt und von der Form der Autonomie durchdrungen ist, scheint eine notwendige Verknüpfung der beiden Sphären ausgeschlossen zu sein, und so ist es in der Tat. Der theoretische Wert des objektiven Sinngebildes bedarf als geltender Wert keiner Verbindung mit dem ethischen Wert des freien Willens. Er ruht in sich und ist sich selbst genug. Er kann insbesondere nicht, wie der über das ethische Gebiet hinausgreifende "Voluntarismus" meint, im Willen sein Fundament haben und diesem seine Würde mitteilen. Das ist mit allem Nachdruck hervorzuheben, damit keine unzulässige Vermengung der Werte entsteht. Aber wir dürfen über der Trennung der Gebiete nicht vergessen, dass zur Totalilät des Theoretischen auch das erkennende Subjekt mit seinem immanenten Sinn gehört, ja dass sogar die wirkliche theoretische Person nicht wegzudenken ist, wenn es sich um die Realisierung der unwirklichen, geltenden Wahrheit in Gütern der Wissenschaft oder wahren Sätzen 
handelt, und damit eröffnet sich uns ein neuer Weg für die Behandlung unseres Problems. Die Verknüpfung des ethischen Wertes mit dem subjektiven Verhalten des theoretischen Menschen ist so wenig ausgeschlossen, dass sie sich vielmehr als notwendig schon aus dem ergibt, was wir über das Wesen der praktischen "Vernunft" gesagt haben. Wir brauchen nur ein gültiges, transzendentes Sinngebilde, in dem Form und Inhalt zusammengehören, auf ein urteilendes Subjekt zu beziehen, das diese Zusammengehörigkeit bejaht, und sofort stellt sich uns das theoretische Subjekt als eine Person ảar, die ein Sollen in ihren Willen aufnimmt. Durch sie allein kommt die Entscheidung zustande, und das Erkennen ist, was immer es als psychischer Vorgang sein mag, seinem immanenten Sinn nach ein Anerkennen des Sollens um des Sollens willen. Die Form wird dem Inhalt beigelegt, weil sie zu ihm gehört. Dem transzendenten Sinn wird zugestimmt, weil er gilt. Der theoretische Wert, der dem Subjekt als Sollen gegenübertritt, ist der zu überwindende "Gegenstand " des Urteilsaktes. Damit kommen wir in das Reich des Ethischen, wie wir es als Wollen des Sollens bestimmt haben. Auch das theoretische Subjekt will autonom, indem es die Wahrheit bejaht, und an seiner Handlung haftet daher der Wert der Freiheit. Zugleich ist die Beziehung des objektiven, transzendenten Sinngebildes auf ein zu ihm Stellung nehmendes Subjekt unvermeidlich, wenn wir das Ganze des theoretischen Gebietes, nicht nur seine sachliche Seite berücksichtigen, und so sind alle Sinn- und Wertbegriffe, die vorher allein für das ethische oder praktische Gebiet charakteristisch zu sein schienen, mit Notwendigkeit in einen Teil des theoretischen Gebiets, nämlich in die subjektive theoretische Sphäre, hineingetragen. Sie können deshalb in dem Ganzen der theoretischen Philosophie auch als gültige Wertbegriffe des immanenten logischen Urteilssinnes nicht entbehrt werden.

Das steht mit den früher hervorgehobenen Gegensätzen vou Sache und Person, Kontemplation und Aktivität, Umschliessen und Durchdringen des Inhalts in keinem Widerspruch. Diese Unterschiede bestehen als Gegensätze nur, solange man begrifflich das Reich der theoretischen Objektivität von dem der praktischen Subjektivität in jeder Hinsicht abscheidet. Das musste im Interesse einer klaren Problemstellung sorgfältig geschehen. Ehe man Begriffe verbindet, sind sie zu trennen. Aber die Trennung darf nicht so verstanden werden, als kämen wir im theoretischen

Kantotudien $\mathbf{X T X}$. 
Gebiet, zu dem das Erkennen der Wahrheit ebenso wie ihre transzendente Geltung gehört, ganz ohne Persönlichkeit und Aktivität aus. Subjekt und Objekt sind notwendig aufeinander angewiesen. Die Person bedarf dei' Sache, um sinnvoll zu wirken, und zur Sache gehört die Person, die sie vertritt. Der Handelnde braucht Kontemplation zur Besinnung auf das, was not tut, und in der Kontemplation, die geltende Werte schöpferisch in sinnvollen Gütern realisieren soll, muss zugleich Aktivität stecken. So liegt es überall, wenn wir den Blick auf das Ganze des sinnvollen Lebens richten, und das theoretische Gebiet ist hiervon nicht ausgenommen. Auch in ihm sind Sache und Person, Kontemplation und Aktivität mit einander verknüpft. Nur vom einseitigen Standpunkt der Sache aus, die transzendent gilt, scheint der theoretische Mensch als blosses Subjekt unpersönlich und passiv die Wahrheit zu finden, und auf die Rechnung seiner Aktivität wird dann das allein gesetzt, was das Wahre in seiner Reinheit trübt. So notwendig aber diese Betrachtungsweise in ihrer besonderen Sphäre sein mag, so wenig kann sie als erschöpfend gelten. Das wirkliche Erkennen muss ebenfalls von der Philosophie verstanden werden, und wenn die Realisierung des theoretischen Wertes in Gütern in Betracht koment, so ist das theoretische Subjekt in seinem immanenten Sinn als Person zu denken, die durch ihr Handeln und Entscheiden zwar gewiss nicht die in sich ruhende und nur aufzufindende Geltung des transzendenten.Sinngebildes, wohl aber das theoretische Gut, an·dem es haftet, erzeugt. Daher stellt dies Subjekt sich notwendig als aktive Persönlichkeit dar, die den Wert der Wahrheit, der ihr als Sollen gegenübertritt, autonom will, und es muss an ihr auch jener ethische Sinn haften, in dem der Inhalt, wie bei allen Sinngebilden der sittlichen Aktivität, von der Form der Freiheit durchdrungen ist.

Vielleicht erscheint das zunächst paradox. Doch handelt es sich im Grunde um einen einfachen Gedanken, und es wird deshalb gut sein, ihn auch ohne die besonderen erkenntnistheoretischen Annahmen und Voraussetzungen, die wir über die Struktur des theoretischen Sinngebildes gemacht haben, noch in einer allgemeineren Formulierung zu geben. Dann können wir sagen: sobald man die Wahrheit als Wert auf einen menschlichen Willen bezieht, werden die wahren Sätze zu Gütern, die sein sollen, und dann muss das theoretische Subjekt als Persönlichkeit gelten, die 
von dem Bewusstsein beherrscht wird, dass sie die Wahrheit zu realisieren habe. Es treibt nur der Mensch Wissenschaft um der Wahrheit willen, der seinem theoretischen Gewissen gehorcht. Daher ist auch er autonom, und an seinem Willen haftet ein Wert, dessen Geltung gerade vom theoretischen Standpunkt aus niemals bezweifelt werden kann. Ja, noch mebr, der pflichtbewusste Wille erweist sich als die metalogische Basis für die Realisierung des theoretischen Gutes, und insofern ist der an ihm haftende Sinn dem theoretischen nicht nur ebenbürtig, sondern muss ihm sogar übergeordnet werden, sobald ausser den Werten auch ihre Verwirklichung in Betracht kommt. Jedenfalls, dass an dem Willen, der will, was er soll, ein Wert haftet, der gilt, kann der Mensch auch als theoretisches Subjekt nicht bezweifeln, da auf diesem Wert der immanente Sinn seines eigenen Denkens und Erkennens beruht. Das ethische Werturteil, das der Autonomie Geltung zuspricht, ist daher von der wissenschaftlichen Philosophie, obwobl es atheoretisch ist, seinem Gehalt nach niemals auf ein Seinsurteil zu reduzieren, sondern sein Anspruch, als Werturteil gültig zu sein, besteht gerade vom theoretischen Standpunkt aus notwendig zu Recht.

Dieser Konsequenz lässt sich nicht dadurch ausweichen, dass man sagt, man wolle ją in der Wissenschaft den Wert der Autonomie nicht bestreiten, sondern meine nur, er gehe als atheoretischer Wert die Wissenschaft überhaupt nichts an. Nur so lange man ausschliesslich bei den Seinswissenschaften bleibt und grundsätzlich jede Wertgeltung als wissenschaftliches Problem ablehnt, ist diese Enthaltsamkeit durchführbar. Treibt man Philosophie als Theorie der Seinswissenschaften und untersucht dabei ihre Geltungsgrundlagen, so darf man den ethischen Wert des autonomen Willens nicht mehr ignorieren, da er zum immanenten Sinn auch des seinswissenschaftlichen Urteilsaktes gehört, und wenn man einmal den Zustand der Indifferenz ihm gegenüber verlassen hat, so muss man seine Geltung, da man sie nicht leugnen kann, ausdrücklich anerkennen. $\mathrm{Ob}$ man dies Werturteil noch in die Logik oder schon in die Ethik rechnen will, darüber kann man vielleicht streiten. Auf jeden Fall aber ist es gültig und gehört in die Philosophie. Diese geht damit in keiner Weise über das wissenschaftliche Gebiet hinaus. Gerade als Wissenschaft von der Wissenschaft darf sic es nicht unterlassen, sich mit dem Sinn des Willens zu beschäftigen, der bei der Urteilsentscheidung oder der Bejahung dor 
Zusammengehörigkeit von Form und Inhalt das will, was er soll, also frei ist.

Dass bei der Behandlung der theoretischen Fragen in einer umfassenden Philosophie nicht allein das objektive, sachliche, kontemplative Gut, sondern ebenso das subjektive, persönliche, aktive Verhalten zu diesem Gut auf seinen Sinn und seine Wertgeltung hin untersucht wird, ist für die Gestaltung der Weltanschauungslehre von geradezu entscheidender Wichtigkeit. Nur auf diesem Wege wird eine einheitliche Deutung unseres Lebenssinnes möglich, welche die beiden grossen Wertsphären in das richtige Verhältnis zueinander bringt, und so wenig wir dies hier in Allgemeinen weiter verfolgen können, so wollen wir doch den damit zusammenhängenden Gedanken, dass atheoretische Probleme innerhalb der theoretischen Philosophie eine Rolle spielen und dadurch zugleich eine unangreifbare theoretische Bedeutsamkeit erhalten, in einer andern Richtung wenigstens andeutend verfolgen, um die prinzipielle Seite der Frage noch mehr hervortreten zu lassen.

Bisher haben wir die Freiheit nur mit Rücksicht auf die Motivation des Wollens als Autonomie behandelt. Gewöhnlich stellt man das Problem so, dass man fragt: kann denn der Wille. auch, was er soll? Hat der Begriff der freien Entscheidung in der Welt der Ursachen überhaupt einen wissenschaftlichen Sinn? So lange man glaubt, dass ethische und theoretische Werte nicht notwendig miteinander verbunden sind, wird man vielleicht meinen, sich unbedenklich für den Determinismus entscheiden zu dürfen, ja, dass es keine Willensfreiheit gibt, erscheint mit Rücksicht auf das Kausalprinzip Vielen geradezu selbstverständlich. Macht man sich dagegen klar, dass auch nach der Freiheit des theoretischen Subjekts gefragt werden muss, falls der immanente Sinn der Erkenntnis verstanden werden soll, dann zeigt sich, dass die deterministische Lösung des Problems der Freiheit in Wahrheit keine Lösung ist, sondern in unüberwindliche Schwierigkeiten führt. Kann, so muss man nun fragen, der Urteilende sich wirklich so entscheiden, wie er sich entscheiden soll? Hat der Begriff der theoretischen Entscheidung überhaupt einen wissenschaftlichen Sinn? Wollte man auch dies Problem mit Hülfe des Determinismus zu lösen versuchen, so würde man die Möglichkeit alles wahren Urteilens, also auch einer deterministischen Entscheidung des Freiheitsproblems aufheben. Jede Leugnung der Freiheit, die wahr sein will, untergräbt ihre eigenen Voraussetzúngen. Schreibt. 
man dagegen dem urteilenden Subjekt die Fähigkeit zu, sich für die Lehren des Determinismus als die wahren zu entscheiden, so ist damit der Determinismus vollends durchbrochen. Die Urteilsfreiheit zu leugnen, ist demnach widersinnig, und dass dies Konsequenzen auch für die Ethik hat, liegt auf der Hand. Oder will man etwa nur die Freiheit, das Wahre zu bejahen, anerkennen, nicht aber die Freiheit, das Gute zu wollen? Es ist nicht einzusehen, wie man diese Meinung konsequent durchzuführen denkt. So wird das Problem der Willensfreiheit in seinem, wenn man will, metaphysisch ethischen Sinn erst für den in seiner ganzen Tragweite überhaupt sichtbar, der die Verknüpfung der theoretischen und der praktischen Probleme und damit die Verknüpfung der lngischen und der ethischen Werte, wie wir sie vorgenommen haben, als wissenschaftlich notwendig verstanden hat.

Doch wir brauchen die Frage nach dieser Seite nicht weiter zu verfolgen. Es kam nur darauf an, zu zeigen, dass auch aus einer rein wissenschaftlichen Philosophie der Wert der Autonomie als gültig nicht $z u$ entfernen ist. Wer sein eigenes Urteilen verstanden hat, muss ihn voraussetzen, und dies ist für unsern Zusammenhang entscheidend. Die Philosophie darf sich nicht einnal als Erkenntnistheorie auf den Standpunkt zurückziehen, dass die Geltung atheoretischer Werte sie nichts angehe, denn das ist gerade ihre Aufgabe, die sie als theoretische Philosophie hat, alle Voraussetzungen der Erkenntnis zum Bewusstsein zu bringen und in Sätzen zu formulieren. Freilich kann man sagen, der autonome Wille ist nur insofern für den wissenschaftlichen Menschen wertvoll, als er Voraussetzung für die Realisierung des theoretischen Gutes ist, aber dies „insofern" schränkt das, worauf es hier ankommt, nicht ein. Wir wollen ja den ethischen Wert nicht auf einen theoretischen zurückführen, sondern zeigen, dass seine Anerkennung, obwohl er atheoretisch bleibt, auch in der Logik nicht vermieden werden kann. Das haben wir getan, und damit ist die Meinung, die Ethik verfahre nicht mehr wissenschaftlich, wenn sie die Geltung dieses Wertes behauptet, im Prinzip abgewiesen. Was die Logik tun muss, das wird auch die Ethik tun dürfen, and was $z \mathfrak{u}$ den Voraussetzungen des theoretischen Gutes der Erkenntnis gehört, ist auch in der Lehre vom sittlichen Handeln über jede unwissenschaftliche Willkür erhaben. 
IX.

Das soziale Individuum.

Nur bis zu diesem Punkt soll der Gedankengang geführt werden. Das Verhältnis der ethischen zur theoretischen Geltung muss jetzt mit Rücksicht auf den denkbar umfassendsten Begriff des sittlichen Wertes klar sein. Trotzdem fügen wir noch eine Andeutung über den nächsten Schritt, den die Ethik wird tun nüssen, und einen flüchtigen Ausblick auf ihre weitere Gestaltung hinzu. Man kann nämlich meinen, dass bis jetzt für die wissenschaftliche Behandlung der sittlichen Güter doch nur wenig oder im Grunde genommen sogar nichts erreicht sei. Freilich, nimmt man den Begriff des Ethischen so umfassend, wie wir es getau haben, dann muss auch der theoretische Mensch darin einen gültigen Wert anerkennen. Zugleich aber scheint dies Ergebnis teuer erkauft. Kann die Ethik als philosophische Sonderdisziplin bei der Lösung ihrer Probleme mit einem so allgemeinen Wert irgend etwas anfangen, ja darf sie ihn noch "ethisch" nennen im Unterschied von andern Werten? Ist hier nicht vielmehr nur ein Begriff gewonnen, der zu den Voraussetzungen jeder Wertverwirklichung gehört? Das, was uns ethisch hiess, ist identisch mit dem autonomen Verhalten gegenüber Werten überhaupt, welches auch immer diese Werte sein mögen, und daher kann dieser Begriff zur Bestimmung der Ethik als einer selbständigen Disziplin im Unterschied von andern Teilen der Wertphilosophie nicht taugen. Das aber wird um so bedenklicher erscheinen, als wir nur von dem weitesten und daher leersten Begriff zeigen kounten, dass er Anerkennung von Seiten des theoretischen Menschen fordert. Die Bestimmung des ethischen Gutes führt uns bei dem Bestreben, ein besonderes Wertgebiet abzustecken, nicht weiter, denn die Persönlichkeit ist in dem bisher angegebenen Sinne auch dann ethisch; wenn sie nur theoretische Werte frei anerkennt. Von ihr aus bekommt also die Ethik als besondere Wissenschaft ebenfalls kein Material, das nicht zugleieh im Begriff des theoretischen Urteilsaktes enthalten ist. Wie wollen wir die Grenze zwischen der theoretischen und der sittlichen Persönlichkeit ziehen? So richtig unsere Darlegungen sein mögen, so unfruchtbar müssen sie sich erweisen, sobald irgend ein Problem des sittlichen Lebens in Angriff genommen wird, das nicht zugleich, wie das autonome Verbalten überhaupt, ein Problem des wissenschaftlichen oder 
des künstlerischen Lebens ist. Wir brauchen einen weniger umfassenden Wert und eine begrenztere Sphäre von Gütern.

In einem gewissen Sinne ist das zutreffend, und jedenfalls darf die Ethik, um ihren Charakter als Wissenschaft zu rechtfertigen, bei dem erreichten Ergebnis nicht stehen bleiben. Sie muss in der Tat den Begriff des autonomen Willens näher bestimmen, um einen engeren Begriff des Ethischen zu gewinnen, und dann die Geltung dieses neuen Wertes ebenfalls als wissenschaftlich unvermeidlich dartun, um ihre Fundamente theoretisch za sichern. Daher weisen wir wenigstens auf das allgemeinste Prinzip, das hier weiter führen kann, zum Schluss noch hin, um seine Beziehungen zu den bis jetzt gewonnenen Begriffen hervortreten zu lassen. Die Autonomie wird vielleicht nicht mehr so leer und unfruchtbar erscheinen, wenn sich zeigen lässt, dass sie mit Notwendigkeit als Form auf ein besonderes Material anzuwenden ist, mit dem zusammen sie dann Sinngebilde von eigner Art ausmacht, und dass diese den Gegenstand der Ethik als einer besonderen philosophischen Disziplin darstellen.

Dabei knüpfen wir wieder an den Begriff des Subjelrts an, da dies der einzige Träger des ethischen Wertes ist. Wir wissen von ihm bisher nur, dass es für die Ethik als Persönlichkeit in Betracht kommit. Doch ist das nicht alles, was wir von ihm sagen können. Als wirkliches Subjekt ist es immer ein Individuum oder ein einmaliges und besonderes Ich, und als solches fordert es nicht nur wie jedes Subjekt ein Objekt als notwendiges Korrelat, sondern zugleich ein bestimmtes Objekt, nämlich ein $\mathrm{Du}, \mathrm{d}$. h. eine andere einmalige und individnelle Persönlichkeit, also eigentlich kein „Objekt", sondern ein anderes Subjekt. Dies $\mathrm{Du}$ als das andere Ich gebört so notwendig zum individuellen Ich wie das Objekt zum Subjekt. Die Person lebt nicht nur faktisch immer in einem $\mathrm{Zu}-$ sammenhang oder in einer Gemeinschaft mit andern Personen, sondern sie lässt sich davon auch begrifflich nicht ablösen. Der Eine ist ohne den Andern nicht zu denken. Die isolierte Persönlichkeit ist eine begriffliche Fiktion, von der wir als dem Begriff des Subjekts vielleicht in der Logik oder in der Ästhetik, nicht aber in der Ethik Gebrauch machen können, da diese es mit den Personen als wirklichen Personen $\mathrm{za}$ tun hat, und es wirkliche Personen nie in der Einzahl, sondern immer nur in der Mebrzahl gibt. Den Zusammenhang dieser Mehrzahl können wir als sozial im weitesten Sinne des Wortes bezeichnen und dann sagen, dass 
das Ich oder die Persönlichkeit, die Träger des ethischen Wertes ist, notwendig ein soziales Ich oder eine soziale Persönlichkeit sein muss. Dementsprechend werden wir auch die Werte und Sinngebilde, die an ihr haften, insofern sie sozial ist, als soziale Werte und soziale Sinngebilde bezeichnen dürfen.

Mit dieser Bestimmung haben wir etwas prinzipiell Wichtiges zunächst insofern gewonnen, als. sie uns gestattet, die früher gegebene allgemeine Einteilung der Wertgebiete zu vervollständigen. Bisher konnten wir nur sagen, dass auf der einen Seite die sachlichen und kontemplativen Güter, wie die theoretischen und die ästhetischen, stehen, und dass ihnen anf der andern Seite die persönlichen und aktiven entsprechen, zu denen die ethischen Güter gehören. Jetzt fügen wir hinzu, dass während die persönlichen und aktiven. Werte in dem angegebenen Sinne sozial sind, die unpersönlichen und kontemplativen, wenigstens in ihrer Reinheit, einen a soziale n Charakter tragen müssen. Das geht schon daraus hervor, dass in dieser Sphäre das Subjekt nicht einmal als Person in Betracht kommt, also vollends der soziale persönliche Zusammenhang der Individuen unwesentlich wird. Freilich ist der Unterschied, da es. sich um die Bedeutung von viel gebrauchten und missbrauchten Schlagworten handelt, ausdrücklich vor Missverständnissen zu . schützen. "Sozial“ darf hier, wie gesagt, nur heissen, dass das ethische Gut, die Persönlichkeit, faktisch stets in irgend einer Gemeinschaft mit anderen Persönlichkeiten lebt und mit Rücksicht auf ihren ethischen Sinn auch begrifflich davon nicht . loszulösen ist. Dementsprechend bedeutet „asozial“ nichts anderes, als dass für die Sinngebilde der theoretischen und der ästhetischen Güter der soziąle Zusammenhang gleichgültig ist. Es soll also damit der Wissenschaft und der Kunst soziale Bedeutung nicht etwa abgesprochen, sondern allein darauf hingewiesen werden, dass die logischen und ästhetischen Werte ohne jede Rücksicht auf den sozialen Zusammenhang der Personen betrachtet werden können, ja in ihrer Reinheit als asozial angeseheu werden müssen.

Besonders ist dann darauf zu achten, dass der so verstandene Unterschied mit denen von Person und Sache, Aktivität und Kontemplation, durchdringender und umschliessender Form notwendig zusammenhängt, ja eigentlich nur eine neue Seite von ihnen beleuchtet, die wir bisher ignoriert haben, um die verschiedenen Probleme begrifflich von einander zu scheiden. Was ich durch 
Kontemplation als Sache vor mich hinstelle, und dessen Inhalt ich theoretisch erkennend oder ästhetisch anschauend mit der Form umschliesse, das muss dadurch, seinem Sinne nach, zu einem von mir abgelösten Objekt werden, kann also auch kein Du bleiben, mit dem ich noch sozial verbunden bin. Und umgekehrt: was seinem Sinne nach ein $\mathrm{Du}$ oder ein anderes Ich ist, mit dem ich in Gemeinschaft lebe, zu dem habe ich notwendig auch irgend ein persönliches und aktives Verhältnis. Das umschliesse ich nicht nur kontemplativ als Sache, sondern in das dringe ich wollend und tätig ein, das ergreife ich, und von dem werde ich ergriffen, denn sonst hätte ich nichts mit ihm "gemein". Das fasse ich mit mir in ein "wir" zusammen, und mit dem setze ich mich daher, gerade indem ich es als $\mathrm{Du}$ von mir unterscheide, zugleich auch in eins, denn wie könnte ich sonst von "wir" und "uns" reden? Und zwar gilt das alles, gleichviel ob ich das andere Ich liebe oder hasse, es unterstütze oder bekämpfe. Das Du nähert sich mir, je mehr ich mich in freundlicher oder feindlicher Gesinnung mit ihm abgebe. Es ist mir das Nächste, was es unter Allem von mir Verschiedenem geben kann, es ist "mein Nächster". Es bildet, zwar nicht in seinem Sein, wohl aber seinem Sin n nach, niemals nur ein anderes Ich, sondern zugleich mein anderes Ich, sodass in der sozialen Sphäre von einer Scheidung in Subjekt und Objekt, wie sie auf dem sachlichen, kontemplativen und asozialen Gebiet zu finden ist, nicht einmal nit Rücksicht auf das andere, mir gegenüberstehende Ich gesprochen werden darf, solange es für mich ein $\mathrm{Du}$ bleibt. Daher muss auch in den. Sinngebilden, die an dem sozialen Verhältnis oder an der Gemeinschaft von Ich und Du haften, die Form den Inhalt durchdringen und nicht nur umschliessen. Vor allem aber: soll diese Form die Autonomie sein, oder habe ich es mit einem sittlich wollenden Du zu tun, so darf ich es niemals nur kontemplativ als Sache vor mich hinstellen, um es als soziales $\mathrm{Du}$ zu vernichten, sondern ich habe es, auch wenn ich es bekämpfe, als Person aktiv zu wollen, ich bin verpflichtet, es als $\mathrm{Du} z \mathrm{zu}$ „behandeln“, also in Gemeinschaft mit ihm zu bleiben. Falls ein anderes Ich für mich nur Sache oder nur Gegenstand der Kontemplation geworden ist, sodass ich mit ihm nicht einmal mehr durch Feindschaftsozial verbunden bin, so kann das nur auf Grund einer vollkommenen ethischen Nichtachtung geschehen, die absolute ethische Sinnlosigkeit der eventuell faktisch noch bestehenden Beziehungen herbeiführt. 
Doch, auch wenn wir vom Ethischen zunächst absehen, so bleibt es jedenfalls dabei: durch Kontemplation und sachliche, umschliessende Form wird jede Gemẹinschaft der Personen zerstört und alles asozial gemacht, während persönliche, eindringende Aktivität nur auf dem Boden eines sozialen Verhältnisses der Individuen $\mathrm{zu}$ einander möglich ist. So verstehen wir, nachdem der Unterschied des Sozialen und des Asozialen hinzugetreten ist, noch besser als früher, warum auf theoretischem und ästhetischem Gebiete das Auseinanderfallen von subjektivem Verhalten und objektivem Gut, auf ethischem Gebiete dagegen ihre Vereinigung herrschend sein muss.

Auf Grund dieser Ausführungen stellen wir dem vorher entwickelten weitesten Begriff des Ethischen als dem des pflichtbewussten Subjekts jetzt den Begriff der sozialen autonomen Persönlichkeit gegenüber und gewinnen damit den Ausblick auf die Möglichkeit, die Ethik als besondere philosophische Disziplin mit eigenem Material aufzubauen. Sie wird Ethik im engeren Sinne, wenn sie die Persönlichkeit nur mit Rücksicht auf die Pflichten behandelt, die ihr als einem Gliede einer Gemeinschaft zukommen, und die sich auf soziale Persönlichkeiten als sozial sinnvolle Güter beziehen. Sie fragt dann weiter notwendig nach Allem, was für die sozialethischen Beziehungen der Persönlichkeiten untereinander Bedeutung hat, iusbesondere welche sozialen Ordnungen als sinnvoll gelten müssen, wenn der ethische Wert der sozialen Persönlichkeit zu seinem Rechte konmen soll. Das ist wichtig, denn damit erweitert sich in einem gewissen Sinn das Gebiet der Ethik über die Persönlichkeiten hinaus, so dass auch Sachen ethisch wesentlich werden. Aber sie sind es immer nur insofern, als von Personen her der ethische Wert und Sinn sich auf sie überträgt, und so bleibt trotz der neuen Bestimmung der Gegensatz von sachlicher, asozialer Kontemplation und persönlicher, sozialer Aktivität unangetastet. Die "sachlichen" oder überpersönlichen ethischen Werte, wie die der Ehe, der Familie, des Rechts,- des Staates, der Nation, der Menschbeit haften immer an Personen als den realen ethischen Gütern und können ohne sie nicht gedacht werden.

Näher brauchen wir dies nicht auszuführen, denn schon jetzt muss klar sein, wie ein Gebiet ethischer Werte und Güter im engeren Sinue des Wortes abzugrenzen ist, und wie also eine philosophische Disziplin entsteht, die sich von der Logik, der 
Ästhetik und andern Teilen der Philosophie auch mit Rücksicht auf ihr Material unterscheidet. Das allein ist hier wesentlich, dass die ethischen Werte an einer Person haften, die bedeatsam ist, insofern sie in einer Gemeinschaft lebt, und dass infolgedessen die Gemeinschaft selbst mit ihren sozialen Institutionen ebenfalls irgendwie ethisch wichtig wird. Beim autonomen Willen des theoretischen Subjektes fällt das Soziale anch in diesem weitesten Sinne fort, denn die theoretischen Sinngebilde gelten in ihrer Reinheit ohne jede Rücksicht auf die sozietas, in der wir leben, und die Pflichten, die wir als rein theoretische Menschen im Dienste der Wahrheit und Wissenschaft haben, sind deshalb nicht mehr "ethische" Pflichten in der jetzt angegebenen engeren Bedeutung. So können wir die Grenze zwischen der sozialen ethischen und der asozialen theoretischen Persönlichkeit scharf ziehen. Die theoretische Persönlichkeit ist zwar ethisch, insofern sie autonon will, aber sie hat mit ihren Urteilsacten lediglich asoziale Güter zu realisieren. Die ethische Persönlichkeit im engeren Sinne handelt nicht nur überhaupt autonom, sondern die Güter, die sie verwirklicht, sind auch begrifflich von den sozialen Zusammenhängen der Persönlichkeiten nicht loszulösen, und dadurch erhalten ihre Pflichten einen besonderen, den sozialethischen Charakter.

Im Übrigen kommt es vor Allem daranf an, dass die Begriffe des Sozialen und der Ethik als Sozialphilosophie vicht zu eng gefasst werden. Besonders ist darauf zu achten; dass das antisoziale Verhalten ebenfalls zu dem in dem angegebenen Sinne sozialen Verhalten gehört. Es ist ja nur gegenüber den Persönlichkeiten, die für uns ein $\mathrm{Du}$ sind, mit denen wir also in irgend einer Gemeinschaft leben, möglich, und auch die antisoziale Handlung greift ein in das andere Ich, stellt es also nicht bloss kontemplativ vor. Das, was man gewöhnlich mit Sozialismus und Individualismus meint, betrifft demnach einen Unterschied, der innerhalb des sozialen Gebietes liegt, und dasselbe gilt vollends von dem Gegensatz der egoistischen und der altruistischen Gesinnung. Deshalb hat in der Ethik als Sozialphilosophie, wie wir sie verstehen, sowohl eine "Individualethik“ als auch eine „Sozialethik ${ }^{\prime}$ im noch engeren Sinne Platz, und es ist ferner keine besondere ethische "Richtung" von vornherein ausgeschlossen. Nicht nur die radikalste Aristokratie bleibt ebenso denkbar wie die radikalste demokratische Tendenz, sondern sogar wenn eine 
auf Grund des Autonomieprinzips ausgeführte Ethik einen radikal antisozialen Charakter trüge, wäre damit unser Begriff der Sozialethik so wenig durchbrochen, wie der Begriff der sozialen Persönlichkeit durch den einer antisozialen Gesinnung, denn eine Theorie, welche diese Gesinnung rechtfertigte, hätte es ebenso wie eine positiv sozial gerichtete mit einer in unserm Sinne sozialen Persönlichkeit zu tun.

Nur das eine wird man fragen, ob eine antisoziale Sozialethik des Autonomieprinzips nicht eventuell dazu kommen könnte, jeden sozialen Zusammenhang der Individuen als ethisch indifferent oder gar als unsittlich anzusehen, und ob daraus dann nicht doch folgen würde, dass der Begriff der sozialen Persönlichkeit in unserem Sinne noch immer nicht bestimmt genug ist, um eine fruchtbare Anwendung auf die Behandlung einzelner ethischer Probleme zu ermöglichen. Aber diese Frage verfolgen wir hier nicht weiter. Ihre Beantwortung gehört in einen anderen Zusammenhang. Dort wäre zu untersuchen, ob so "unwiderleglich“ selbstverständlich das antisoziale Gewissen als atheorethisches Gewissen ist, die Ethik, gerade falls sie Wissenschaft sein will, jemals in die Lage kommen kann, ein antisoziales Gewissen theoretisch $\mathrm{zu}$ rechtfertigen, oder ob nicht vielmehr das seinem rein logischen Wesen nach asoziale Denken, wenn es einmal seine soziale Indifferenz verlassen, nach dem Wert des Sozialen gefragt hat und dadurch auf die Gesellschaft bezogen ist, auch den Begriff der Gültigkeit zu dem der "Allgemeingültigkeit" als einer sozialen Gültigkeit gestalten muss, sodass dann vom theoretischen Denken her in die wissenschaftliche Behandlung der sozialen ethischen Probleme notwendig ein positives soziales Moment hineingetragen würde. Mit anderen Worten: verwandelt sich in der ethischen Theorie vom sozialen Individuum das unpersönliche, asoziale Subjekt, in dessen Namen die Wissenschaft sonst als "wir" redet, nicht insofern in ein persönliches, soziales Subjekt, als gerade das wissenschaftliche "wir" seinem Begriff nach niemals antisozial werden kann? Von dieser Frage aus liesse sich die Verknüpfung theoretischer und ethischer Werte und die.Möglichkeit einer theoretischen Begründung auch des Sozialethischen weiter verfolgen. Man müsste untersuchen, 0 b) der autonome Wille und das positive soziale Gewissen etwa einander ausschliessen, insofern jede Rücksicht auf den "Anderen" Heteronomie bedeutet, oder ob sie zwar verträglich sind, ohne jedoch in einer notwendigen Beziehung zu stehen, 
oder endlich, ob sie sich vielleicht als wissenschaftlich notwendig miteinander verknüpft erweisen, wenn der wollende Mensch auf sein Sollen theoretisch reflektiert, so dass vom Standpunkt der wissenschaftlichen Ethik soziale Werte auch im positiven Sinn als ethisch notwendig zu fordern sind. Hier beschränken wir uns, wie gesagt, auf die Verbindung des theoretischen Wertes mit dem allgemeinsten und daher leersten Werte der Autonomie und weisen nur darauf hin, dass es auf jeden Fall die weitere Aufgabe der Ethik ist, dies formale Prinzip auf die sozialen Zusammenhänge der Individuen irgendwie anzuwenden, wobei dann als das Material, an dem die einzelnen Probleme zu finden sind, vor Allem die geschichtliche Mannigfaltigkeit des sozialen Lebens in Betracht kommt. Diese Andeutung soll den Verdacht zurückweisen, als könne sich die Ethik auf das Prinzip der Autonomie in seiner umfassendsten Bedeutung beschränken. In der Logik ist ja ebenfalls nicht nur von der Wahrheit im Allgemeinen, sondern auch von ihrer besonderen Ausgestaltung in der Wissenschaft zu handeln. Für die wissenschaftliche Erforschung des autonomen Lebens gilt nicht minder, dass sie eine inhaltliche Erfüllung braucht, die aus den formalen Grundwerten nicht zu deduzieren ist. Doch wollten wir hier gerade zeigen, dass trotzdem schon durch den weitesten, form̧alen Begriff der Autonomie eine Brücke zwischen Wahrheit und Sittlichkeit geschlagen und so die allgemeinste werttheoretische Grundlage der Ethik wissenschaftlich befestigt wird. 